One has to reckon on the luck of finding the desired TS in the allowed region. In contrast, the empirical MINDO-type procedures are universal and well suited for dealing with equilibrium molecular geometries, but their extrapolation into the intermolecular interaction region can result in very serious errors. The need for a reliable semiempirical method to treat chemical reactions is still far from being exhausted.

\section{References and Notes}

(1) P. D. Bartlett, Q. Rev., Chem. Soc., 24, 473 (1970).

(2) W. von Doering, M. Franck-Neumann, D. Hasselmann, and R. L. Kaye, J. Am. Chem. Soc., 94, 3833 (1972).

(3) M. Taagepera and E. R. Thornton, J. Am. Chem. Soc., 94, 1168 (1972)

(4) J. R. McCabe and C. A. Eckert, Acc. Chem. Res., 7, 251 (1974)

(5) L. M. Stephenson, R. V. Gemmer, and S. Current, J. Am. Chem. Soc., 97, 5909 (1975).

(6) M. J. S. Dewar, "The Molecular Orbital Theory of Organic Chemistry", McGraw-Hill, New York, N.Y., 1969

(7) R. B. Woodward and R. Hoffmann, "The Conservation of Orbital Symmetry", Academic Press, New York, N.Y., 1970.

(8) M. V. Basilevsky, Adv. Chem. Phys., 33, 345 (1975).
(9) D. Rowley and H. Stein, Discuss. Faraday Soc., 10, 198 (1951).

(10) L. Salem, J. Am. Chem. Soc., 90, 553 (1968).

(11) O. Klkuchi, Tetrahedron, 27, 2791 (1971).

(12) M. V. Basilevsky, V. A. Tikhomirov, and I. E. Chlenov, Theor. Chim. Acta, 23, 75 (1971).

(13) M. V. Basilevsky and V. A. Tikhomirov, Theor. Eksp. Khim., 8, 723 (1972).

(14) M. J. S. Dewar, A. C. Griffin, and S. Kirschner, J. Am. Chem. Soc., 96, 6225 (1974).

(15) J. W. Mclver, Jr., J. Am. Chem. Soc., 94, 4782 (1972).

(16) (a) L. A. Burke, G Leroy, and M Sana, Theor. Chim. Acta, 40, 313 (1975): (b) L. A. Burke and G. Leroy, ibid., in press.

(17) R. E. Townshend, G. Ramunni, G. Segal, W. J. Hehre, and L. Salem, J. Am Chem. Soc., 98, 2190 (1976).

(18) R. J. Boyd and M. A. Whitehead, J. Chem. Soc., Dalton Trans, 1, 73 (1972).

(19) M. V. Basilevsky and V. A. Tikhomirov, Int. Congr. Pure Appl. Chem., 23rd 1, 109 (1971)

(20) R. C. Bingham, M. J. S. Dewar, and D. H. Lo, J. Am. Chem. Soc., 97, 1285 (1975).

(21) M. J. S. Dewar and E, Haselbach, J. Am. Chem. Soc., 92, 590 (1970).

(22) J. R. Hoyland, Theor. Chim. Acta, 22, 229 (1971).

(23) The invariance is slightly disturbed because an empirical parametrization of one-center exchange integrals cannot exactly reproduce the transformational properties of AO's. However, we have agreed to neglect these integrals.

\title{
Proton-Electron Hyperfine Coupling Constants of the Chlorophyll $a$ Cation Radical by ENDOR Spectroscopy
}

\author{
H. Scheer, J. J. Katz,* and J. R. Norris \\ Contribution from the Chemistry Division, Argonne National Laboratory, \\ Argonne, Illinois 60439. Received April 12, 1976
}

\begin{abstract}
In this paper we describe the assignment of the major coupling constants in monomer chlorophyll $a$ cation free radical by ENDOR spectroscopy. To facilitate chemical manipulation methylpyrochlorophyllide $a$ has been used as a stand-in, and a suite of six selectively deuterated derivatives have been subjected to ENDOR investigation. Details of the synthesis of these compounds are described. To study the effect of structural features on the spin distribution in the free radicals, six additional chlorophyll derivatives have been studied. Five coupling constants have been assigned, which account for about $80 \%$ of the observed electron spin resonance line width in the chlorophyll $a$ monomer cation radical. The spin distribution appears to be highly asymmetric.
\end{abstract}

ENDOR $^{1}$ spectroscopy has provided important support ${ }^{2-8}$ for the original ESR interpretation ${ }^{9-11}$ that the primary donor of photosynthesis contains two special chlorophylls. Detailed comparisons of in vivo and in vitro ENDOR data have provided an experimental basis for a variety of special pair chlorophyll models ${ }^{9,12-24}$ as the primary donor in photosynthesis. In all previous ENDOR studies an interpretation of the spin density distribution in terms of a specific molecular structure has not been documented. For conclusions based on the in vivo ENDOR to be the most convincing, the ENDOR spectrum should account for the whole of the unpaired spin density rather than reflect only a small fraction of an electron distribution. Incomplete interpretation is a likely possibility since all sites of spin density are not necessarily observable by ENDOR spectroscopy. ${ }^{25}$ Thus a molecular basis for assignment of the ENDOR spectra of the chlorophyll cations is important.

In this paper we report the detailed analysis of the protonENDOR spectrum of the cation radical of chlorophyll $a(1)$, methylpyrochlorophyllide $a(9)$, and of a series of related magnesium chlorins. These data provide a clear description of specific assignments of the electron-proton hyperfine coupling constants of in vitro chlorophyll $a \pi$ cation free radical and provide firm support for the view that the ENDOR spectrum of the Chl.+ free radical accounts essentially for all of the protons on the chlorophyll macrocycle. Comparisons between in vivo and in vitro ENDOR data therefore do in fact appear to be essentially complete.

The model compound (9) is essentially identical to chlorophyll $a$ insofar as spin distribution in the cation free radicals is concerned, but it offers a much greater variety of possibilities for structural modifications and for the selective introduction of deuterium. The results of the ENDOR spectroscopy on a wide range of selectively deuterated and structurally modified chlorins establish that chlorophyll $a \pi$ cation free radical has low spin density at the four methine bridges and the nitrogen atoms of the pyrrole rings. Although the results are in general agreement with open shell calculations for chlorins, the chlorophyll $a$ cation radical shows a strongly asymmetric spin distribution that cannot as yet be completely accounted for by current theory. Finally we show experimentally that this asymmetry is the result of ring $\mathrm{V}$ of the chlorophyll macrocycle and is not a result of other various functional side groups.

\section{Experimental Section}

Spectra. ENDOR spectra were recorded on a Varian E-700 spectrometer with a Varian large access cavity and at a temperature of $100 \mathrm{~K}$ on samples prepared on the vacuum line. Compounds $(0.5-1$ $\mathrm{mg}$ ) were first dried by dissolution in benzene, followed by evaporation 
Chart I

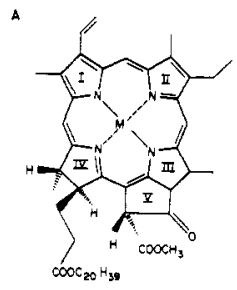

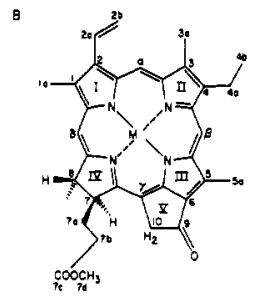

$\underline{2}, \underline{9}$

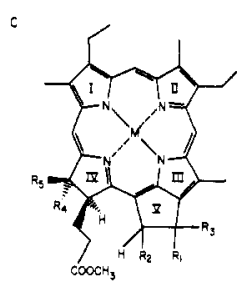

$3,4.8$

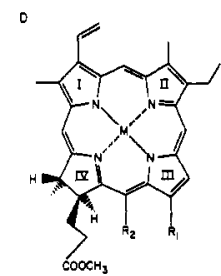

$5,6,7$
$13,14,15$

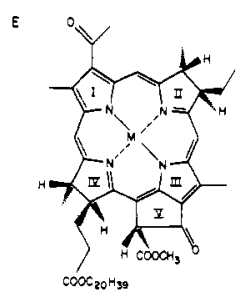

$\underline{16}$

\begin{tabular}{|c|c|c|c|c|c|c|c|}
\hline \multicolumn{2}{|c|}{ Compd No ${ }^{a}$} & \multirow{2}{*}{\multicolumn{4}{|c|}{ Modifications }} & \multirow[b]{3}{*}{ Remarks } & \multirow[b]{3}{*}{ Location } \\
\hline \multirow{2}{*}{$\begin{array}{l}\text { Free basec } \\
\left(\mathrm{M}=\mathrm{H}_{2}\right)\end{array}$} & \multirow{2}{*}{$\begin{array}{l}\text { Chlorophyllide } \\
(\mathrm{M}=\mathrm{Mg})\end{array}$} & & & & & & \\
\hline & & $\mathrm{R}_{1}, \mathrm{R}_{3} b$ & $\mathrm{R}_{2}$ & $\mathrm{R}_{4}$ & $\mathrm{R}_{3}$ & & \\
\hline & $1,1 \mathrm{a}$ & & & & & Chlorophyll $a$ & A \\
\hline $2,2 \mathrm{a}, 2 \mathrm{~b}, 2 \mathrm{c}$ & $9,9 a, 9 b$ & & & & & $\begin{array}{l}\text { Basic Chl a } \\
\text { derivative }\end{array}$ & $\mathrm{B}$ \\
\hline $3,3 \mathrm{a}$ & $12,12 \mathrm{a}, 12 \mathrm{~b}, 12 \mathrm{c}$ & $=0$ & $\mathrm{H}$ & $\mathrm{CH}_{3}$ & $\mathrm{H}$ & & $\mathrm{C}$ \\
\hline 4 & 10 & $\mathrm{H}_{2}$ & $\mathrm{H}$ & $\mathrm{CH}_{3}$ & $\mathrm{H}$ & Racemic & $\mathrm{C}$ \\
\hline 5 & 14 & $\mathrm{COOCH}_{3}$ & $\mathrm{CH}_{2} \mathrm{COOCH}_{3}$ & & & No ring $V$, no $R_{3}$ & $\mathrm{D}$ \\
\hline 6 & 15 & $\mathrm{H}$ & $\mathrm{CH}_{2} \mathrm{COOCH}_{3}$ & & & No ring $V$, no $R_{3}$ & $\mathrm{D}$ \\
\hline 7 & 13 & $\mathrm{COOCH}_{3}$ & $\mathrm{H}$ & & & No ring $V$, no $R_{3}$ & $\mathrm{D}$ \\
\hline 8 & 11 & $=\mathrm{O}$ & $\mathrm{H}$ & $\mathrm{H}$ & $\mathrm{CH}_{3}$ & Racemic & $\mathrm{C}$ \\
\hline & 16 & & & & & Bacteriochlor ophyll & $\mathrm{E}$ \\
\hline & 17 & $=0$ & $\mathrm{OH}$ & $\mathrm{CH}_{3}$ & $\mathrm{H}$ & C- 10 epimers & $\mathrm{C}$ \\
\hline
\end{tabular}

$a_{1} a, 2 a, 2 b$, etc., refer to the corresponding compound with isotopic alterations. ${ }^{b}$ Compounds $5,6,7,13,14$, and 15 contain no $\mathrm{R}_{3}$ group. ${ }^{c}$ ' In the free base $\mathrm{Mg}$ has been replaced by $\mathrm{H}_{2}$.

to dryness at $60^{\circ} \mathrm{C}$ in a stream of $\mathrm{N}_{2}$. Additional drying was accomplished by codistillation with methylene chloride and/or cyclohexane and heating under high vacuum. The sample was then dissolved in 0.75 $\mathrm{ml}$ of $\mathrm{C}^{2} \mathrm{H}_{2} \mathrm{Cl}_{2}$ and $0.25 \mathrm{ml}$ of $\mathrm{C}^{2} \mathrm{H}_{3} \mathrm{O}^{2} \mathrm{H}$, and successive small portions of iodine were added until an optimal ESR signal was obtained. The evacuated sample was then sealed off and stored at $77 \mathrm{~K}$. Chemical oxidation was used to generate chlorin cation free radicals because light cannot be readily introduced into this Varian ENDOR cavity. The free radicals produced by chemical oxidation have been shown to be identical with those produced by photoexcitation. ${ }^{10}$

Electron excitation spectra (UV-visible) were recorded on a Cary 14 RI spectrometer. The extinction coefficient of methylpyrochlorophyllide $a(9)$ was measured directly, and those of all the other $\mathrm{Mg}$ chlorins were determined indirectly by the following method: One milligram of the compound was dissolved in $10 \mathrm{ml}$ of diethyl ether and the spectrum was recorded. The solution was shaken with $5 \mathrm{ml}$ of 2 $\mathrm{N} \mathrm{HCl}$ and washed with $\mathrm{NaHCO}_{3}$, the ethereal solution was evaporated to dryness, and the residue was redissolved in $10 \mathrm{ml}$ of a suitable solvent. A spectrum of the thus formed free chlorin was recorded and the extinction coefficient $(\epsilon)$ of the original Mg-containing compound was calculated relative to the literature value of $\epsilon$ of the free chlorin. With methylpyrochlorophyllide $a(9)$ this procedure gave results identical within experimental error with those obtained by conventional procedures.

NMR spectra were recorded on a Varian HR 220 spectrometer operated in the pulse-FT mode. Chemical shifts are given in $\delta(\mathrm{ppm})$ relative to TMS or HMS as internal standard. In the exchange experiments, the isotopic compositions were determined by calibration against the signal of the NH proton(s). These are completely exchanged during work-up. Mass spectra were obtained on an AEI MS-9 spectrometer. Mg analysis was carried out by atomic absorption spectroscopy. Sample solutions were obtained by demetalation of the $\mathrm{Mg}$ chlorins dissolved in $\mathrm{CH}_{2} \mathrm{Cl}_{2}$, and the $\mathrm{Mg}^{2+}$ was extracted into water by three extractions with $0.5 \mathrm{~N}$ hydrochloric acid.

General Conditions. All solvents were reagent grade. Peroxides were removed by chromatography over basic alumina (Woelm). Magnesium perchlorate (G. F. Smith) was dried for $6 \mathrm{~h}$ at $225^{\circ} \mathrm{C}$ at $p \leqslant 10^{-5}$ Torr. "Work-up" of the preparations consisted of thorough washing of the organic phase with water (if acidic, first with $\mathrm{NaHCO}_{3}$ solution), drying with $\mathrm{NaCl}$, and evaporation under vacuum. The preparative TLC of the free bases were carried out on $20 \times 20 \mathrm{~cm}$ plates, coated with $0.75 \mathrm{~mm}$ Adsorbosil 5 (Applied Science Laboratories), loaded with $10 \mathrm{mg}$ of the crude product. The chlorophyllides were chromatographed on powdered sugar columns or by HPLC (Waters Associates).

Compounds. See Chart I for structures of numbered compounds.

Chlorophyll $a(1)$, deuteriochlorophyll $a\left((1)-{ }^{2} \mathrm{H}\right)$, and methylpyropheophorbide $a(2)$ were obtained by standard procedures. ${ }^{26,27}$ Deuterated methylpyropheophorbide $a\left((2)-{ }^{2} \mathrm{H}\right)$ was obtained from 1- ${ }^{2} \mathrm{H}$ by carrying out the transesterification to methylpheophorbide $a$ in ${ }^{2} \mathrm{HCl} / \mathrm{C}^{2} \mathrm{H}_{3} \mathrm{O}^{2} \mathrm{H}$ and the subsequent pyrolysis in pyridine containing ${ }^{2} \mathrm{H}_{2} \mathrm{O}$.

'H NMR shows 0.4 protons at position $10,0.14$ protons at position $5 \mathrm{a}$, and 0.14 protons in the $\delta$ position. meso-Methylpyropheophorbide $a$ (3) was obtained from 2 by catalytic hydrogenation, ${ }^{28}$ and rac-3 and rac-9-deoxo-meso-methylpyropheophorbide $a(4)$ by reaction of 2 under Wolff-Kishner conditions. ${ }^{29}$ Compounds with an open isocyclic ring, chlorine $\mathrm{e}_{6}$ trimethyl ester (5), isochlorin $\mathrm{e}_{4}$ dimethyl ester (6), and rhodochlorin dimethyl ester (7) were obtained by suitable modifications of the methods of Fischer. ${ }^{30,31}$ 7,8-cis-mesoMethylpyropheophorbide $a(8)$ was obtained by Krasnovskii reduction of zinc phylloerythrin. ${ }^{32}$

Selective Labeling of Methylpyropheophorbide a (2). (a) $10-\mathrm{C}^{2} \mathrm{H}_{2}$ : $20 \mathrm{mg}$ of 2 was refluxed for $48 \mathrm{~h}$ under $\mathrm{N}_{2}$ in $1 \mathrm{ml}$ of pyridine- ${ }^{2} \mathrm{H}_{5} / 2 \%$ ${ }^{2} \mathrm{H}_{2} \mathrm{O}$. Work-up and TLC on silica $95: 5 \mathrm{CCl}_{4} /$ acetone yielded $18 \mathrm{mg}$ $(90 \%)$ of the $10-\mathrm{C}^{2} \mathrm{H}_{2}$ compound. UV-visible and TLC were identical with that of $2 ;{ }^{1} \mathrm{H}$ NMR showed no $10-\mathrm{CH}_{2}$ signal $(<5 \%)$. Under the same conditions, (2) $-{ }^{2} \mathrm{H}$ yielded with protic reagents a compound that shows by ' $\mathrm{H}$ NMR $2.2 \mathrm{H}$ at position $10,0.26 \mathrm{H}$ at position $\delta$, and 0.8 $\mathrm{H}$ at position $5 \mathrm{a}$.

(b) $5-\mathrm{C}^{2} \mathrm{H}_{3}, 10-\mathrm{C}^{2} \mathrm{H}_{2}$ : according to the procedure of Inhoffen and Mengler ${ }^{33} 50 \mathrm{mg}$ of 2 was refluxed for 14 days under $\mathrm{N}_{2}$ in $2 \mathrm{ml}$ of pyridine- ${ }^{2} \mathrm{H}_{5} / 2 \%{ }^{2} \mathrm{H}_{2} \mathrm{O}$. Work-up and TLC on silica yielded $42 \mathrm{mg}$ (84\%) of the $5-\mathrm{C}^{2} \mathrm{H}_{3}, 10-\mathrm{C}^{2} \mathrm{H}_{2}$ compound. UV-visible and TLC were identical with that of 2 ; ' $\mathrm{H}$ NMR showed no $10-\mathrm{CH}_{2}(<5 \%)$ and $5-\mathrm{CH}_{3}(<10 \%)$. Under the same conditions, $(2)-{ }^{2} \mathrm{H}$ yielded with protic reagents a compound which shows by 'H NMR $2.1 \mathrm{H}$ at position 10 , $0.24 \mathrm{H}$ at position $\delta$, and $2.3 \mathrm{H}$ at position 5 .

(c) $\delta-{ }^{2} \mathrm{H}, 7^{\prime \prime}-\mathrm{C}^{2} \mathrm{H}_{3}: 20 \mathrm{mg}$ of 2 was refluxed for $1 \mathrm{~h}$ with $1 \mathrm{ml}$ of $\mathrm{C}^{2} \mathrm{H}_{3} \mathrm{O}^{2} \mathrm{H} / 5 \%{ }^{2} \mathrm{H}_{2} \mathrm{SO}_{4}$. Work-up and TLC yielded $17 \mathrm{mg}(85 \%)$ of the $\delta-{ }^{2} \mathrm{H}, 7-\mathrm{C}^{2} \mathrm{H}_{3}$ compound. UV-visible and TLC were identical with that of $2 ;{ }^{\prime} \mathrm{H}$ NMR showed no $\delta-\mathrm{H}$ or $7^{\prime \prime}-\mathrm{CH}_{3}$ signal $(<5 \%)$. Under the same conditions, $(2)-{ }^{2} \mathrm{H}$ yielded with protic reagents a compound that shows $3.1 \mathrm{H}$ at position $7^{\prime \prime}, 1.7 \mathrm{H}$ at position 10 , and $0.9 \mathrm{H}$ at the methine position $\delta$ by ${ }^{~} \mathrm{H}$ NMR.

Selective Deuteration of rac-meso-Methylpyropheophorbide a (3). 


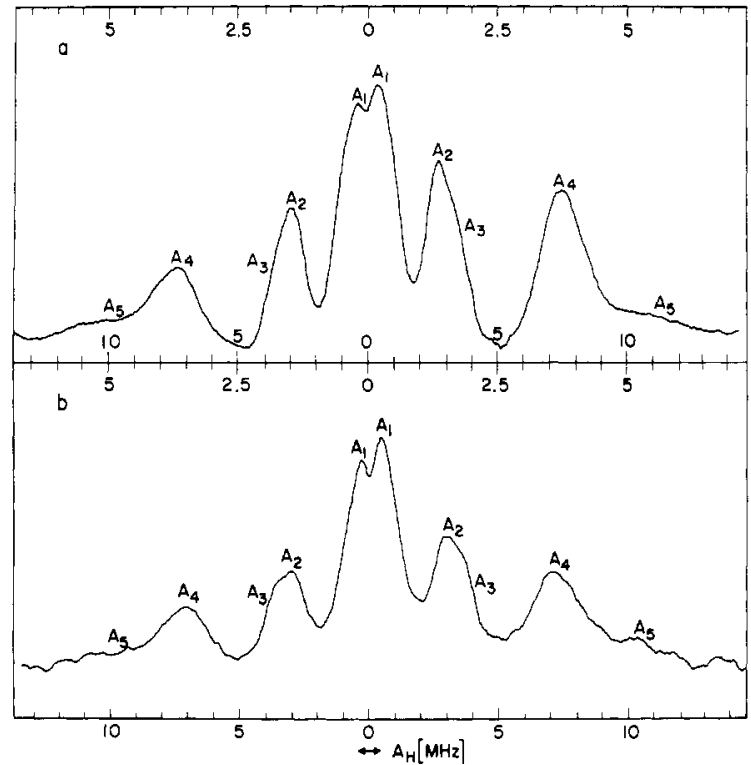

Figure 1. Comparison of (a) ENDOR of chlorophyll $a$ (1) with (b) ENDOR of methylpyrochlorophyllide $a$ (9). Lower scale of each spectrum is labeled such that coupling constants of each peak $A$ can be read directly in $\mathrm{MHz}$. Upper scale of each spectrum is labeled in true units of $\mathrm{MHz}$ frequency shift from the free proton resonance value. In other words one unit of the upper scale corresponds to two units of the lower scale.

Isotopically substituted 3 was prepared by a modification of the method of Fischer and Gibian. $.^{29}(2)-{ }^{2} \mathrm{H}(19 \mathrm{mg})$ was dissolved in 0.5 $\mathrm{ml}$ of pyridine and $2 \mathrm{ml}$ of a freshly prepared saturated solution of sodium methoxide in methanol and $2 \mathrm{ml}$ of $85 \%$ hydrazine hydrate was added. The mixture was purged with $\mathrm{N}_{2}$ and heated in a sealed ampule to $100^{\circ} \mathrm{C}$ for $8 \mathrm{~h}$. The crude product was hydrolyzed for $1 \mathrm{~h}$ with $25 \mathrm{ml}$ of $25 \% \mathrm{HCl}$ at $100^{\circ} \mathrm{C}$ and the chlorin was then extracted with ether after dilution of the mixture to $2 \% \mathrm{HCl}$. Refluxing for 1 $h$ with $\mathrm{C}^{2} \mathrm{H}_{3} \mathrm{O}^{2} \mathrm{H} / 5 \%{ }^{2} \mathrm{H}_{2} \mathrm{SO}_{4}$ yielded the methyl esters, which after work-up were separated by TLC on silica $\left(95: 5 \mathrm{CCl}_{4}\right.$ /acetone). Labeled rac-meso-methylpyropheophorbide $a(3)$ was obtained in $26 \%$ yield, the main by-product being the corresponding 9-desoxo compound (4) (18\%). UV-visible and TLC of the labeled 3 and of its methylpheophorbide were identical with those of an authentic sample. 'H'NMR: 9.39 (s, $\beta) ; 9.12(\mathrm{~s}, \alpha) ; 8.38(\mathrm{~s}, \delta) ; 5.21,5.03$ (AB pattern, $\left.J=18 \mathrm{~Hz}, 10-\mathrm{CH}_{2}\right) ; 4.36(\mathrm{~s}, 8-\mathrm{H}) ; 4.18(\mathrm{~s}, 7-\mathrm{H}) ; 3.58,3.53,3.22$ (aromatic $\left.\mathrm{CH}_{3}\right) ; 2.50,2.18(\mathrm{AB}, J=16 \mathrm{~Hz}, 7 \mathrm{~b}-\mathrm{H})$.

Insertion of Magnesium. A solution of $5 \mathrm{mg}$ of the free base chlorin in $2 \mathrm{ml}$ of pyridine/ $1 \%$ water is heated under $\mathrm{N}_{2}$. After reflux begins $300 \mathrm{mg}$ of dry magnesium perchlorate is added at once. The refluxing is continued, and the metalation reaction is followed spectrophotometrically to completion (2-10 $\mathrm{min}$ ). The slurry obtained after cooling was extracted with pyridine and separated by centrifugation. The pyridine extract (1 part) was then partitioned against a mixture of petroleum ether ( 1 part), water ( 1 part), and methylene chloride $(0.2$ parts).

The upper phase was washed four times with water, dried with $\mathrm{NaCl}$, and evaporated. Any residual water and pyridine is removed by codistillation with benzene at $60^{\circ} \mathrm{C}$. The residue is then dissolved in dry benzene and chromatographed. After the free base chlorin is washed off the column with dry benzene, the $\mathrm{Mg}$ complex is eluted with benzene $/ 0.5 \% 1$-propanol, washed with water, and dried. The yield of metalated product is about $80 \%$.

Methylpyrochlorophyllide a (9). UV-visible (ether): $\lambda_{\max }\left(\epsilon \times 10^{-3}\right)$ $=659$ (71.0), 613 (11.2), 428 (104), 407 (63.3), $382 \mathrm{~nm}$ (39.8). Mg analysis: found, $4.75 \%$; calcd, $4.13 \%$; ${ }^{1} \mathrm{H}$ NMR, IR, and TLC identical with an authentic sample.

rac-trans-9-Deoxo-meso-methylpyrochlorophyllide a (10). UVvisible (ether): $\lambda_{\max }\left(\epsilon \times 10^{-3}\right)=617(70.6), 570(6.1), 406(354), 386$ (100)

rac-7,8-cis-meso-Methylpyrochlorophyllide a (11). UV-visible (ether): $\lambda_{\max }\left(\epsilon \times 10^{-3}\right)=651(92.3), 611(12.0), 603(11.6), 572$ (6..1), 436 (121), 405 (73.6), $380 \mathrm{~nm}(41.0)$. UV-visible (pyridine): $\lambda_{\max }\left(\epsilon \times 10^{-3}\right)=659(67.0), 631(12.9), 608(10.6), 437(100.5)$, $415(58.7), 393 \mathrm{~nm}(33.1) .{ }^{1} \mathrm{H}$ NMR: $\delta[\mathrm{ppm}]$ in acetone ${ }^{2} \mathrm{H}_{6}=9.55$ $(\mathrm{s}, \beta) ; 9.12(\mathrm{~s}, \alpha) ; 8.29(\mathrm{~s}, \delta) ; 5.00,4.95\left(\mathrm{dd}, 10-\mathrm{H}_{2}\right) ; 4.75(\mathrm{~m}, 7-\mathrm{H})$; $4.62(\mathrm{~m}, 8-\mathrm{H}) ; 3.76,3.73\left(\mathrm{q}, 2,4-\mathrm{CH}_{2}\right) ; 3.53\left(\mathrm{~s}, 7 \mathrm{~d}-\mathrm{CH}_{3}\right) ; 3.33,3.25$, $3.17\left(\mathrm{~s}, 1,3,5-\mathrm{CH}_{3}\right) ; 2.57\left(\mathrm{~m}, 7 \mathrm{a}-\mathrm{CH}_{2}\right) ; 2.15\left(\mathrm{~d}, 8-\mathrm{CH}_{3}\right) ; 1.68(\mathrm{t}$, $\left.2 \mathrm{a}, 4 \mathrm{a}-\mathrm{CH}_{3}\right) ; \delta(\mathrm{ppm})$ in pyridine ${ }^{2} \mathrm{H}_{5}=9.82(\mathrm{~s}, \beta) ; 9.28(\mathrm{~s}, \alpha) ; 8.40$ $(\mathrm{s}, \delta) ; 5.225 .12\left(\mathrm{dd}, 10-\mathrm{H}_{2}\right) ; 4.69(\mathrm{q}, 8-\mathrm{H}) ; 4.42(\mathrm{~m}, 7-\mathrm{H}) ; 3.68,3.65$ $\left(\mathrm{q}, 2,4-\mathrm{CH}_{2}\right) ; 3.70\left(7 \mathrm{~d}-\mathrm{CH}_{3}\right) ; 3.26,3.12,3.05\left(1,3,5-\mathrm{CH}_{3}\right) ; 2.41$ $\left(7 \mathrm{a}-\mathrm{CH}_{2}\right) ; 1: 93\left(\mathrm{~d}, 8-\mathrm{CH}_{3}\right) ; 1.61,1.55\left(\mathrm{t}, 2 \mathrm{a}, 4 \mathrm{a}-\mathrm{CH}_{3}\right)$.

7,8-trans-meso-Methylpyrochlorophyllide a (12). UV-visible (ether): $\lambda_{\max }\left(\epsilon \times 10^{-3}\right)=647(95.1), 607(12.4), 598(11.6), 567(5.9), 423$ (136), 403 (78.9), $378 \mathrm{~nm}$ (41.9). UV-visible (pyridine): $\lambda_{\max }(\epsilon \times$ $\left.10^{-3}\right)=656(74.0), 630(13.2), 607(11.1), 436(114), 412(72.8), 393$ $\mathrm{nm}(43.5) .{ }^{1} \mathbf{H}$ NMR: $\delta(\mathrm{ppm})$ in acetone ${ }^{2} \mathrm{H}_{6}=9.51(\mathrm{~s}, \beta) ; 9.07(\mathrm{~s}$, $\alpha) ; 8.25(\mathrm{~s}, \delta) ; 4.44(\mathrm{q}, 8-\mathrm{H}) ; 4.18(\mathrm{~m}, 7-\mathrm{H}) ; 3.69,3.63\left(\mathrm{q}, 2,4-\mathrm{CH}_{2}\right)$; $3.63\left(7 \mathrm{~d}-\mathrm{CH}_{3}\right) ; 3.47,3.11$ (s, aromatic $\left.\mathrm{CH}_{3}\right) ; \sim 2.3\left(\mathrm{~m}, 7-\mathrm{CH}_{2} \mathrm{CH}_{2}\right)$; $1.59\left(\mathrm{~d}, 8-\mathrm{CH}_{3}\right) ; 1.51,1.47\left(\mathrm{t}, 2,4-\mathrm{CH}_{3}\right)$.

Rhodochlorin Dimethyl Ester Magnesium (13). UV-visible (ether): $\lambda_{\max }\left(\epsilon \times 10^{-3}\right)=646(87.0), 598(11.8), 414(257), 395 \mathrm{~nm}(90.4)$ UV-visible (pyridine): $\lambda_{\max }\left(\epsilon \times 10^{-3}\right)=654(83.2), 617(17.3), 430$ (198), $409 \mathrm{~nm}(93.8)$. Mg analysis: calcd, $4.12 \%$; found, $3.82 \% .{ }^{1} \mathbf{H}$ NMR: $9.69,9.55,9.53,8.99(\mathrm{~s}, \alpha, \beta, \gamma, \delta) ; 6.54,5.98$ (vinyl $\mathrm{H}_{\mathrm{A}}, \mathrm{H}_{\mathrm{B}}$ ), $4.27,3.78,3.55,3.38,3.32\left(\mathrm{~s}\right.$, aromatic and ester $\left.\mathrm{CH}_{3}\right), 3.60\left(4-\mathrm{CH}_{2}\right)$, $1.71\left(\mathrm{t}, 4-\mathrm{CH}_{3}\right), 1.69\left(\mathrm{~d}, 8-\mathrm{CH}_{3}\right)$.

Chlorin $e_{6}$ Trimethyl Ester Magnesium (14). UV-visible (ether): $\lambda_{\max }$ $\left(\epsilon \times 10^{-3}\right)=642(76.0), 597(11.3), 522(6.8), 414(203), 395 \mathrm{~nm}$ (96.0). UV-visible (pyridine): $\lambda_{\max }\left(\epsilon \times 10^{-3}\right)=649(66.0), 616(13.7)$, 430 (208), $408 \mathrm{~nm}$ (86.5). Mg analysis: calcd, 3.68\%; found, 3.20\%. 'H NMR: $9.58,9.55,8.38(\mathrm{~s}, \alpha, \beta, \delta), 5.34\left(\mathrm{~s}, \gamma-\mathrm{CH}_{2}\right), 7.88,6.26,6.00$ (vinyl $\mathrm{H}_{\mathrm{X}}, \mathrm{H}_{\mathrm{A}}, \mathrm{H}_{\mathrm{B}}$ ), 3.76, 3.61, 3.45, 3.36, 3.33 (s, aromatic and ester $\left.\mathrm{CH}_{3}\right), 1.67\left(\mathrm{t}, 4-\mathrm{CH}_{3}\right), 3.76\left(\mathrm{q}, 4-\mathrm{CH}_{2}\right), 1.50\left(\mathrm{~d}, 8-\mathrm{CH}_{3}\right)$.

Isochlorin $\mathbf{e}_{\mathbf{4}}$ Dimethyl Ester Magnesium (15). UV-visible(ether): $\lambda_{\max }\left(\epsilon \times 10^{-3}\right)=635(91.6), 591(11.3), 412(277), 393 \mathrm{~nm}(106)$. UV-visible (pyridine): $\lambda_{\max }=642,607,427,409 \mathrm{~nm}$. Relative intensities = 1:0.18:3.64:1.31. Mg Analysis: calcd, 4.03\%; found, 3.97\%.

\section{Results and Discussion}

The Model Compound. The choice of a model compound for the selective deuteration experiments was dictated by two principal factors: (a) chlorophyll $a$ itself (1) is unstable toward acids, bases, and heat, and (b) the demetalated chlorophyll $a$ (cf. pheophytin $a$ ), although in principle much easier to modify chemically, cannot as yet be remetalated in reasonable yields. The model compound which overcomes both problems is methylpyrochlorophyllide $a(9)$. The chemistry of the metalfree methylpyropheophorbide $a(2)$ has been thoroughly investigated, and, because the enolizable $\beta$-ketoester system is lacking, the compound can be smoothly remetalated to the chlorophyllide. ${ }^{34,35}$ In methylpyrochlorophyllide $a(9)$ the propionic acid phytylester has been transesterified with methanol, and the 10-carbomethoxy group has been removed by pyrolysis. While the propionic ester is several carbon atoms away from the $\pi$ system and thus is not expected to couple noticeably with the free electron, this is not necessarily true for the changes which result from the removal of the 10-carbomethoxy group and the addition of an additional new $\beta$ proton (ESR terminology) at $\mathrm{C}-10$. The coupling constant of the 10-protons is very small (see below), however, and thus the substitution of $\mathrm{H}$ for $\mathrm{COOCH}_{3}$ has negligible influence on the ENDOR spectrum. The ENDOR spectra of chlorophyll $a$ (1) and methylpyrochlorophyllide $a(9)$ are virtually indistinguishable (Figure 1), and this establishes methylpyrochlorophyllide (9) as an appropriate model compound for chlorophyll $a$ (1).

Insertion of Magnesium. Magnesium, like most divalent metals, is easily incorporated into porphyrins, ${ }^{36}$ but its insertion into pheophorbides has always been a problem. ${ }^{37}$ As demonstrated recently, the resistance to $\mathrm{Mg}$ insertion is not related to the reduced 7,8 double bond or the isocyclic ring but rather is to be attributed to the enolizable $\beta$-ketoester system present in most chlorophylls and their derivatives. ${ }^{34}$ Thus, the metal is easily incorporated into methylpyropheophorbide $a$ (2) which lacks the $\beta$-ketoester system by the refluxing with anhydrous $\mathrm{Mg}\left(\mathrm{ClO}_{4}\right)$ (or other $\mathrm{Mg}$ salts) in pyridine. The re- 
Chart II. Reaction Scheme for Selective Deuteration and Derivative Preparations from Methylpyropheophorbide $a$ (2)
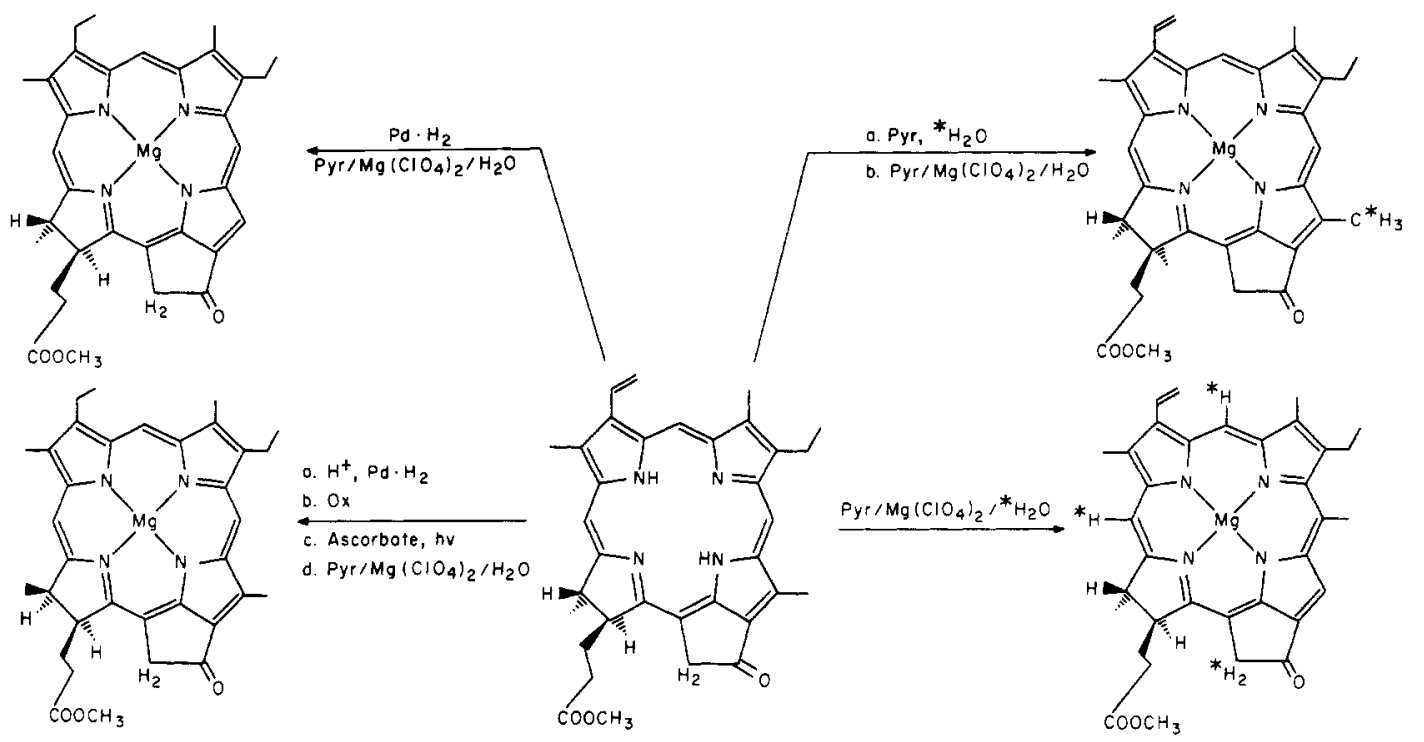
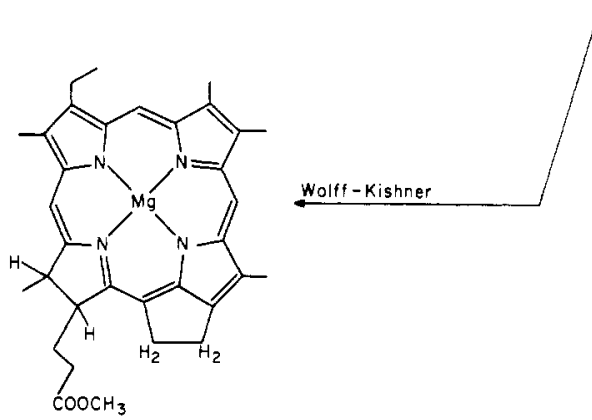

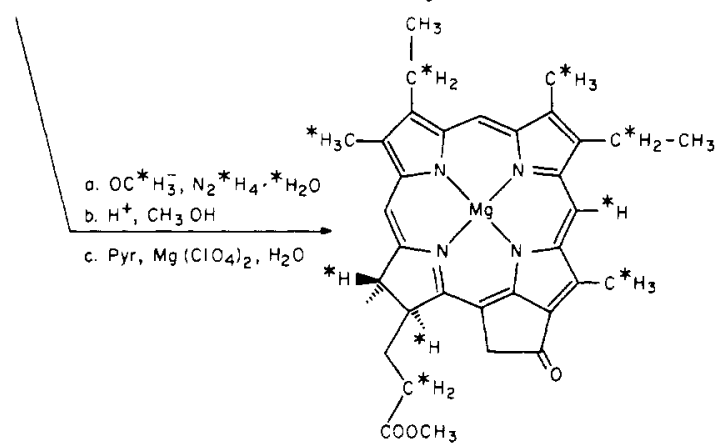

action can be followed spectrophotometrically, the best spectral region for this being between 550 and $470 \mathrm{~nm}$, where the two overlapping intermediate intense bands $(\epsilon \sim 12000)^{38}$ disappear upon complexation. Magnesium insertion is markedly accelerated by water. Under reasonably available anhydrous conditions ( $\sim 0.1 \mathrm{~mol} \mathrm{H}_{2} \mathrm{O} / \mathrm{l}$.), methylpyropheophorbide $a$ is metalated to $90 \%$ within $1.5 \mathrm{~h}$. Gradual increase of the water concentration to $\sim 0.6 \mathrm{M}$ decreases this time to 5-10 min, and the reaction is virtually complete within $15 \mathrm{~min}$.

The $\mathrm{Mg}$ complex is partially purified during the work-up procedure, the final separation from by-products and the pheophorbide adduct being achieved by chromatography on powdered sugar. In contrast to the less polar chlorophylls and pheophytins, the $7 \mathrm{~d}$ methyl esters are considerably more strongly adsorbed. Thus, the columns can be more highly loaded, and a benzene-propanol mixture is suitable for elution.

During the metalation reaction, not only the 10 protons but also the methine protons are exchanged. This observation is paralleled by results of Smith et al., ${ }^{40}$ who reported the unexpected exchange of the methine hydrogens in porphyrins during metalation with $\mathrm{Mg}$. Both the accelerated $\mathrm{Mg}$ insertion and the isotope exchange are possibly due to acid catalysis by water. The $\delta$ and 10-protons are easily exchangeable under acidic conditions, but the $\alpha$ - and $\beta$-methine protons are observed to exchange usually only at very low $\mathrm{pH} .{ }^{37,41}$ There are indications, however, of a considerably increased acidity of water bound to $\mathrm{Mg}^{2+}$ in the pyridine $/ \mathrm{Mg}\left(\mathrm{ClO}_{4}\right)_{2}$ system. At low water concentrations, the water ${ }^{1} \mathrm{H}$ NMR resonance is shifted to very low field $(\delta>10 \mathrm{ppm})$, and with increasing amounts of water the signal shifts back to its usual value in pyridine at 5 ppm. ${ }^{42}$ Such a low-field shift is characteristic for acidic protons.

Selective Deuterium Exchange (See Chart II) and Additional
Model Compounds. As a general rule, all benzylic protons in pheophorbides are exchangeable under basic conditions. However, the rates of exchange show differences distinct enough to allow selective labeling at least for some of them. See Chart II. In Chl $a$ (1) the 10-proton is easily exchanged, being part of the enolizable $\beta$-ketoester system. ${ }^{43}$ In methylpyropheophorbide (2), this exchange occurs in refluxing pyridine with traces of water, via enolization of the 9-CO group. ${ }^{44}$ Prolonged refluxing under these conditions leads to a selective exchange of the $5-\mathrm{CH}_{3}$ protons as well, which can be rationalized by a vinologous enolization mechanism. ${ }^{33}$ Under the highly basic conditions of the Wolff-Kishner reduction, ${ }^{29}$ all benzylic positions as well as the enolic $7 b$-protons are exchanged. As shown by mirror image experiments (see Experimental Section), the C-3 and C-5 methyl protons, the 10 methylene protons, the methine protons, the "chlorin" protons at $\mathrm{C}-7$ and $\mathrm{C}-8$, and the enolic $7 \mathrm{~b}$-methylene protons are completely exchanged under these experimental conditions, while the $1-\mathrm{CH}_{3}$ as well as the 2- and $4-\mathrm{CH}_{2}$ protons are only partially exchanged. This reaction also results in racemization at C-7 and C-8, and in reduction of the 2-vinyl group. Under acidic conditions, the $\delta$-H is substituted ${ }^{41}$ and the 7 -propionic acid side chain can be transesterified. ${ }^{30}$ All these exchange experiments have been carried out both by starting from ' $\mathrm{H}$-methylpyropheophorbide $a$ and exchange with deuterium and by starting with the fully deuterated compound and exchanging with protons. The latter approach is of considerable advantage in the assignment of ENDOR lines otherwise hidden.

As mentioned above, the final insertion of magnesium provides a further possibility of exchange (or reexchange) of some of the hydrogen atoms. In this reaction, the $10-\mathrm{CH}_{2}$ protons and the $\alpha$ - and $\delta$-methine protons are simultaneously exchanged, which poses limitations on some of the selective ex- 
Table I. ENDOR Proton-Electron Coupling Constants (MHz) of Magnesium Chlorins

\begin{tabular}{|c|c|c|c|c|c|c|c|}
\hline & Compd & $\mathbf{A}_{1}$ & $\mathrm{~A}_{2}$ & $\mathrm{~A}_{3}$ & $\mathrm{~A}_{4}$ & $\mathrm{~A}_{5}$ & Remarks \\
\hline $\begin{array}{l}(1) \\
(9)\end{array}$ & $\begin{array}{l}\text { Chlorophyll } a \\
\text { Methylpyrochlorophyllide } a\end{array}$ & 0.76 & 2.83 & 3.72 & 7.56 & 11 & \\
\hline$(9 a)$ & Normal isotopic composition & 0.81 & 3.02 & 3.81 & 7.22 & 10.9 & \\
\hline $\begin{array}{l}(9 b) \\
(9 c)\end{array}$ & $\begin{array}{l}5-\mathrm{C}^{2} \mathrm{H}_{3}, 10-\mathrm{C}^{2} \mathrm{H}_{2} \\
\text { Only } \alpha-\mathrm{H}, \delta-\mathrm{H}, 10-\mathrm{CH}_{2}\end{array}$ & $\begin{array}{c}\text { n.v. } \\
0.5 / 1.67(\mathrm{sh})\end{array}$ & 2.83 & 3.50 & & $7-10$ & Protic solvent \\
\hline (12) & $\begin{array}{l}\text { 7,8-trans- } \\
\text { meso-Methylpyrochlorophyllide } a\end{array}$ & & & & & & \\
\hline $\begin{array}{l}(12 a) \\
(12 b) \\
(12 c)\end{array}$ & $\begin{array}{l}\text { Normal isotopic composition } \\
3,5-\mathrm{C}^{2} \mathrm{H}_{3}, 7,8-{ }^{2} \mathrm{H} \\
3,5-\mathrm{C}^{2} \mathrm{H}_{3}, 7,8-{ }^{-} \mathrm{H} \text { only }\end{array}$ & $\begin{array}{l}0.65 \\
0.62 \\
0.26\end{array}$ & $\begin{array}{l}2.77 \\
3.0 \\
2.40\end{array}$ & $\begin{array}{l}3.69 \\
2.92\end{array}$ & $\begin{array}{l}7.25 \\
7.06\end{array}$ & $\begin{array}{l}11.1 \\
10\end{array}$ & $\begin{array}{l}\text { 2-Vinyl missing } \\
1-\mathrm{CH}_{3}, 2,4-\mathrm{CH}_{2} \text {, part exch. } \\
1-\mathrm{CH}_{3}, 2,4-\mathrm{CH}_{2} \text {, part exch. }\end{array}$ \\
\hline $\begin{array}{l}\text { (11) } \\
\text { (10) }\end{array}$ & $\begin{array}{l}\text { 7,8-cis- } \\
\text { meso-Methylpyrochlorophyllide } a \\
\text { 9-Deoxo-meso- }\end{array}$ & 0.57 & 2.52 & 3.35 & 6.45 & $8 / 19.8$ & \\
\hline $\begin{array}{l}(17) \\
(14) \\
(13) \\
(15)\end{array}$ & $\begin{array}{l}\text { methylpyrochlorophyllide } a \\
\text { 10-Hydroxychlorophyll } a \\
\text { Chlorin } \mathrm{e}_{6}-\mathrm{TME}-\mathrm{Mg} \\
\text { Rhodochlorin-DME-Mg } \\
\text { Isochlorin } \mathrm{e}_{4}-\mathrm{DME}-\mathrm{Mg}\end{array}$ & $\begin{array}{c}1.44 \\
\text { n.v. } \\
0.36 / 0.84 \\
0.44(\mathrm{sh}), 0.98 \\
0.44(\mathrm{sh}), 0.86\end{array}$ & $\begin{array}{l}2.91 \\
3.30 \\
3.00 \\
2.77 \\
3.16\end{array}$ & $\begin{array}{l}3.54 \\
3.91\end{array}$ & $\begin{array}{l}8.26 \\
7.34 \\
3.57 \\
4.69\end{array}$ & $\begin{array}{l}11.5 \\
\text { n.v. } \\
\sim 7.20 \\
8(?) \\
7.69\end{array}$ & $\begin{array}{l}\text { No 9-CO, 2-vinyl } \\
\text { Protic solvent } \\
\text { Ring V opened } \\
\text { No } \gamma \text { substituent } \\
\text { No } 6 \text { substituent }\end{array}$ \\
\hline
\end{tabular}

change experiments with respect to the final $\mathrm{Mg}$ complexes. The isotopic composition of the final products (Table I) has been established from their ' $\mathrm{H}$ NMR spectra, in which all signals in question are well separated and fully assigned. ${ }^{45}$

To determine the influence of the various substituents on the proton-electron coupling constants, several additional structural modifications were made on the model compound 2. These compounds are tabulated in Chart I. The mesopheophorbide (3) was obtained by hydrogenation of the 2-vinyl group, ${ }^{28}$ the 9-carbonyl group in the 9-deoxopheophorbide (4) was removed by Wolff-Kishner reduction, ${ }^{29}$ and the 7,8-cispheophorbide (8) was obtained by Krasnovskii reduction. ${ }^{32}$ The influence of the isocyclic ring was studied with compounds in which ring $\mathrm{V}$ has been opened, viz., the magnesium complexes of chlorin $e_{6}$ trimethyl ester (14), isochlorin $e_{4}$ dimethyl ester (15), and rhodochlorin dimethyl ester (13) (Chart I).

The ENDOR Spectrum of Chlorophyll a and Methylpyrochlorophyllide a. The ENDOR spectrum of the cation radical of Chl $a$ (Figure 1a, Table I) shows three well resolved bands $\left(A_{1}, A_{2}, A_{4}\right)$, a shoulder $\left(A_{3}\right)$ at the $A_{2}$ band, and a broad band $\left(\mathrm{A}_{5}\right)$ partially hidden by $\mathrm{A}_{4}$ and extending as far as $7 \mathrm{MHz}$. The spectrum is reasonably symmetric with respect to the free proton frequency, indicating no contributions from couplings with nuclei other than protons in the frequency range studied. However, different intensities for the respective high- and low-frequency ENDOR lines are often encountered. These variations in intensity not only arise because of the operation of different relaxation mechanisms but involve also a nonlinear rf sweep, the exact position of the magnetic field $H_{0}$ with respect to the ESR transition, and perhaps other factors. The ESR spectrum is very similar in dry aggregating and disaggregating solvents which indicates a negligible spin delocalization in chlorophyll oligomers formed by keto $\mathrm{C}=\mathrm{O} \cdots \mathrm{Mg}$ interactions. ${ }^{9}$ The spectrum shown in Figure 1 is typical for deuterated or, more precisely, ${ }^{1} \mathrm{H}$-free solvents. ${ }^{1} \mathrm{H}$-containing solvents give rise to a pronounced matrix peak ${ }^{25}$ at $A \sim 0 \mathrm{MHz}$, which usually obscures the $A_{1}$ peaks, and which arises from residual coupling of the free electron with solvent molecules.

The ENDOR spectrum of the cation radical of methylpyrochlorophyllide $a(9)$ (Figure lb) is essentially identical with that of Chl $a *^{+}$. This indicates not only a negligible coupling constant for an unpaired electron with the remote protons of the esterifying alcohol at $\mathrm{C}-7 \mathrm{~b}$, but also suggests a very small coupling constant and low intensity for the C-10 proton(s) coupling constant.
Aromatic Methyl and Methylene Coupling Constants. ${ }^{1} \mathrm{H} /{ }^{2} \mathrm{H}$ exchange of the 5-methyl group (compound 9b) results in the loss of the major ENDOR line $A_{4}$, with only the underlying broad resonance corresponding to the shoulder $\mathrm{A}_{5}$ in the spectrum of 9 remaining in the spectrum. Obviously, this major ENDOR line in 9 represents coupling only with a single methyl group at C-5. The favorable cross-relaxation time of rotating methyl groups as compared to the more rigid $\alpha$-protons was first described by Hyde et al. ${ }^{25}$ For frozen solutions, intense bell-shaped lines were predicted and experimentally verified. The ENDOR spectra of both Chl $a(1)$ and Bchl $a$ (16) cation radicals are each dominated by two such lines, and for 16 it can indeed be shown by biosynthetic insertion of deuterium that these peaks arise from the methyl groups in positions 1 and $5 .{ }^{46,47}$ The assignment of the $A_{4}$ line to the 5- $\mathrm{CH}_{3}$ group makes it very probable that the similarly shaped $\mathrm{A}_{2}$ line likewise originates from interactions involving other methyl groups. In contrast to $\mathbf{1 6}, \mathbf{1}$ has one additional aromatic methyl group at position $\mathrm{C}-3$. This suggests that both the 1and $3-\mathrm{CH}_{3}$ resonances of $\mathrm{Chl} a^{+}{ }^{+}$are contained in the second (methyl) ENDOR line $A_{2}$. This assignment is supported by the relative intensities of $\mathrm{A}_{4}$ and $\mathrm{A}_{2}$. In $\mathrm{Bchl} a^{+}{ }^{+}$both lines represent electron couplings with one methyl group each. The line corresponding to the larger coupling, which we assign by analogy with $\mathrm{Chl} a^{+}$to the $5-\mathrm{CH}_{3}$ coupling, is about twice as intense as the $1-\mathrm{CH}_{3}$ ENDOR line. This is a reflection of the general rule that, given similar relaxation, the intensity of an ENDOR line increases with the magnitude of the coupling constants. In $\mathrm{Chl} \mathrm{a}^{+}$, the ENDOR line, $\mathrm{A}_{2}$, corresponding to the smaller coupling is increased about twofold for intensity and becomes comparable in size to $\mathrm{A}_{4}$, thus supporting the assignment of one methyl group (5-- $\left.\mathrm{CH}_{3}\right)$ to $\mathrm{A}_{4}$ and two methyl groups (1- and $3-\mathrm{CH}_{3}$ ) to $\mathrm{A}_{2}$.

This assignment is further supported by ${ }^{2} \mathrm{H}$ exchange experiments of 12 . In compound $12 \mathrm{~b}$, the only $\beta$ type protons that are not completely exchanged are those of the partially exchanged 2- and 4- $\mathrm{CH}_{2}$ and the 1- $\mathrm{CH}_{3}$ groups. The ENDOR spectrum of $12 \mathrm{~b}$ shows a single line corresponding to $\mathrm{A}_{2}$ in the ENDOR spectra of compounds 1,9 , and 12 . It can be concluded from this experiment that not only the 1- and $3-\mathrm{CH}_{3}$ protons but also the 4- $\mathrm{CH}_{2}$ protons give rise to the $\mathrm{A}_{2}$ ENDOR line and the $\mathrm{A}_{3}$ shoulder as well. Assuming comparable spin density distributions in $\mathbf{9}$ and 12, this further indicates that the shoulder $\mathrm{A}_{3}$ arises from coupling with the $3-\mathrm{CH}_{3}$ protons. These conclusions are again supported by the corresponding mirror image experiment. Exchange of ${ }^{2} \mathrm{H}$ by ${ }^{1} \mathrm{H}$ (in compound 
(12) $-{ }^{2} \mathrm{H}$ ) leads to a pronounced diminuation of the $\mathrm{A}_{2}$ resonance in the ENDOR spectrum and the $A_{3}$ wing (as compared to the $\mathrm{A}_{4}$ resonance), corresponding to the only partial exchange of the $1-\mathrm{CH}_{3}, 2-\mathrm{CH}_{2}$, and $4-\mathrm{CH}_{2}$ deuterons under the exchange conditions.

Methine Protons. Exchange of the $\delta$ - and $\beta$-methine protons and the $10-\mathrm{CH}_{2}$ protons with ${ }^{2} \mathrm{H}$ causes no significant changes in the ENDOR spectrum. Obviously, none of the major lines are related to couplings with these protons. Direct information about coupling with protons in these positions can be obtained, however, from (mirror) isotope inversion studies. Compound $9 c$, which contains protons only in the $\alpha, \delta$, and 10 positions (the latter only to the extent of about $50 \%$ ), gives rise to a single unresolved ENDOR line corresponding to $A \approx 0.5 \mathrm{MHz}$ with a shoulder at $A=1.67 \mathrm{MHz}$. All three of these sites may contribute to the $\mathrm{A}_{1}$ line in chlorophyll $a$. During the $\mathrm{Mg}$ insertion step all three positions are exchanged simultaneously and without pronounced discrimination, which precludes further discrimination between these resonances from exchange experiments alone. As indicated below, the shoulder on $\mathrm{A}_{1}$ probably arises from the $\mathrm{C}-10$ protons. The $\alpha$ - and $\delta$-methine interactions are then assigned to the $A=0.5 \mathrm{MHz}$ ENDOR line. A small coupling constant involving the third methine proton, i.e., $\beta$-H, is indicated by an exchange experiment with ${ }^{2} \mathrm{H}$-chlorophyll. Occupation of the $\beta, \delta$, and $10-\mathrm{H}$ positions can be effected by a reversible (Krasnovskii) photoreduction with $\mathrm{H}_{2} \mathrm{~S} .{ }^{48}$ The ENDOR spectrum of ${ }^{2} \mathrm{H}$-chlorophyll in which $\beta, \delta$, and 10 positions are occupied by ${ }^{1} \mathrm{H}$ shows only a single center peak corresponding to couplings $\leqslant 0.5$ $\mathrm{MHz}$.

7,8-Protons. An unambiguous assignment of the 7,8-proton couplings is not possible only from selective deuteration experiments. The 7,8-protons can be exchanged under the strongly basic conditions of the Wolff-Kishner reduction, but under these conditions most of the benzylic protons $(1,3,5-$ $\mathrm{CH}_{3}$, 4- and $10-\mathrm{CH}_{2}$ ) are exchanged as well, thus precluding a sufficiently selective deuteration. In $12 b$, the $A_{4}$ ENDOR line is missing, and the intensity of $A_{2}$ as compared to $A_{1}$ is diminished, which as mentioned above aided in the assignment of the latter resonance to interactions with methyl groups. The spectrum of this compound in addition lacks the broad resonance $A_{5}$. This resonance is present and unchanged in all other modified chlorophyllides except for one, which indicates that it does not belong to the vinyl, C-10, or methine protons, and thus an assignment to the 7,8-protons suggested itself. This assignment is supported by the spectrum of compound 11 (Figure 2a), which is the only chlorophyllide whose ENDOR spectrum shows a pronounced change in the $A_{5}$ signal. Compound 11 is a stereoisomer of the meso-chlorophyllide (12), in which the 7 and 8 protons are cisoid to each other ${ }^{32}$ in contradistinction to the transoid configuration present in chlorophylls. ${ }^{29.45 .49-51}$ The ${ }^{1} \mathrm{H}$ NMR spectrum of 11 indicates pronounced conformational changes in ring IV, while the ${ }^{1} \mathrm{H}$ NMR resonances of the remaining protons in the molecules are less perturbed (cf. ref 32). In the ENDOR spectrum of 11 the most pronounced change is the appearance of a broad resonance with the unusually large coupling constant of $A=$ 19.9 $\mathrm{MHz}$. This large coupling leads to a doublet in the ESR spectrum, and indeed the cation radical of $\mathbf{1 1}$ is the only one of the compounds investigated with an intact ring $\mathrm{V}$ that shows any ESR fine structure at all. The coupling constant obtained from the ESR spectrum $(A \approx 20 \mathrm{MHz})$ agrees well with that observed by ENDOR, and the multiplicity of the signal proves interaction with one proton to be responsible for the coupling. As the pronounced configurational and conformational changes of ring $\mathrm{IV}^{32}$ must be responsible for this new coupling, we assign it to one of the "extra hydrogens" at C-7 and C-8. This strongly suggests that the similarly large and broadened $\mathrm{A}_{5}$ resonance in the 7,8-trans-chlorophyllides is due as well to coupling with these protons.

The assignment of the largest coupling constant in $\mathrm{Chl} \mathrm{a}^{+}$ to the "extra" hydrogens at C-7 and C-8 is paralleled by a similar assignment in the cation radical of bacteriochlorophyll $a(16) .^{52}$ The ESR spectrum of magnesium tetraphenylbacteriochlorin.+ ${ }^{+}$, which was used as a model compound for $\mathbf{1 6}$ on the basis of spectroscopic similarities, shows a clearly resolved $7.5 \mathrm{G},(21 \mathrm{MHz})$ coupling with the eight equivalent "chlorin" protons of the reduced peripheral bonds.

2-Vinyl and 10-Protons. The vinyl group in chlorophylls has been shown to be partially conjugated, but not coplanar with the aromatic macrocycle by ${ }^{1} \mathrm{H} \mathrm{NMR}^{45}$ and $\mathrm{UV}$-visible spectroscopy. The vinyl protons are thus intermediate in coupling between $\alpha$ - and $\beta$-protons. In a fully coplanar conformation, the only coupling mechanism would be spin polarization by spin transfer to the vinyl groups. With increasing dihedral angle of the 2-2a bond a hyperconjugative coupling can be expected to occur as well. For intermediate angles both effects are expected to be small, but as the protons in the $1-\mathrm{CH}_{3}$ group have a large coupling constant, a substantial coupling with the 2 a proton could be possible by both mechanisms. Selective exchange of the vinyl protons was not possible in a straightforward way, but the spin density at the 2-position can be estimated from the meso compound (12). In 12, the 2-vinyl group is converted to a 2 -ethyl group by hydrogenation, and consequently two true $\beta$-protons are introduced into the molecule. This leads in the ENDOR spectrum to an additional shoulder with a smaller $A$ value than the $\mathrm{A}_{2}$ resonance, while the remainder of the spectrum is for the most part unchanged. We, therefore, suggest that the shoulder on $\mathrm{A}_{2}$ arises from interactions with the $2-\mathrm{CH}_{2}$ protons in 12 . Although spin redistribution upon hydrogenation of the 2-vinyl group cannot be excluded, the negligible changes in other parts of the spectrum indicate that the new signal does indeed arise from a new group, rather than from change in the coupling constants giving rise to $\mathrm{A}_{2}$. This assignment is reasonable, as a similar spin density is expected for both C-1 and C-2. In addition, it would suggest a similar coupling constant for the $4-\mathrm{CH}_{2}$ protons, which would likewise be concealed in the unresolved $\mathrm{A}_{2}$ ENDOR peak. The only other significant difference in the ENDOR spectra of 9 and $\mathbf{1 2}$ is the coupling constant of the $A_{1}$ ENDOR line. This line is a superposition of several unresolved couplings, and $A_{1}$ thus represents a mean value only. The pronounced increase $(0.38$ vs. $0.65 \mathrm{MHz})$ on conversion of the 2-vinyl group in 1 to a 2-ethyl group in 12 indicates a dominant component with very small coupling in 1 . We take this as indication for a value of $A \leqslant 0.38 \mathrm{MHz}$ for coupling by the vinyl protons, although no distinction between the three vinyl protons can be made in this way.

The coupling constant of the 10-proton(s) is probably small, or its relaxation behavior is so unfavorable that the ENDOR signal is too small to be detected. This may be concluded from the similarity of the spectra of methylpyrochlorophyllide $a(9)$ and of $\mathrm{Chl} a$ (1). In the former compound, C-10 is substituted with two protons, while $\mathrm{Chl} a$ itself bears only one at this position.

The cation radicals of both compounds have virtually identical ENDOR spectra, which excludes a major contribution of these protons to the ENDOR spectrum. The coupling constant of the 10 -protons is more directly accessible from a mirror isotope experiment. In compound 9c, the $\alpha-, \beta$-, and 10 -positions are the only ones bearing ${ }^{1} \mathrm{H}$, while all other hydrogens in the molecule are ${ }^{2} \mathrm{H}$. The ENDOR spectrum shows two lines, a weak one with $A=1.67 \mathrm{MHz}$ and a stronger one with $A=0.5 \mathrm{MHz}$. As discussed above, exchange occurs simultaneously in all three positions during the metalation procedure, and these three protons cannot be differentiated. An assignment of $10-\mathrm{H}$ to the larger coupling constant with low intensity is probable, however, from the following con- 

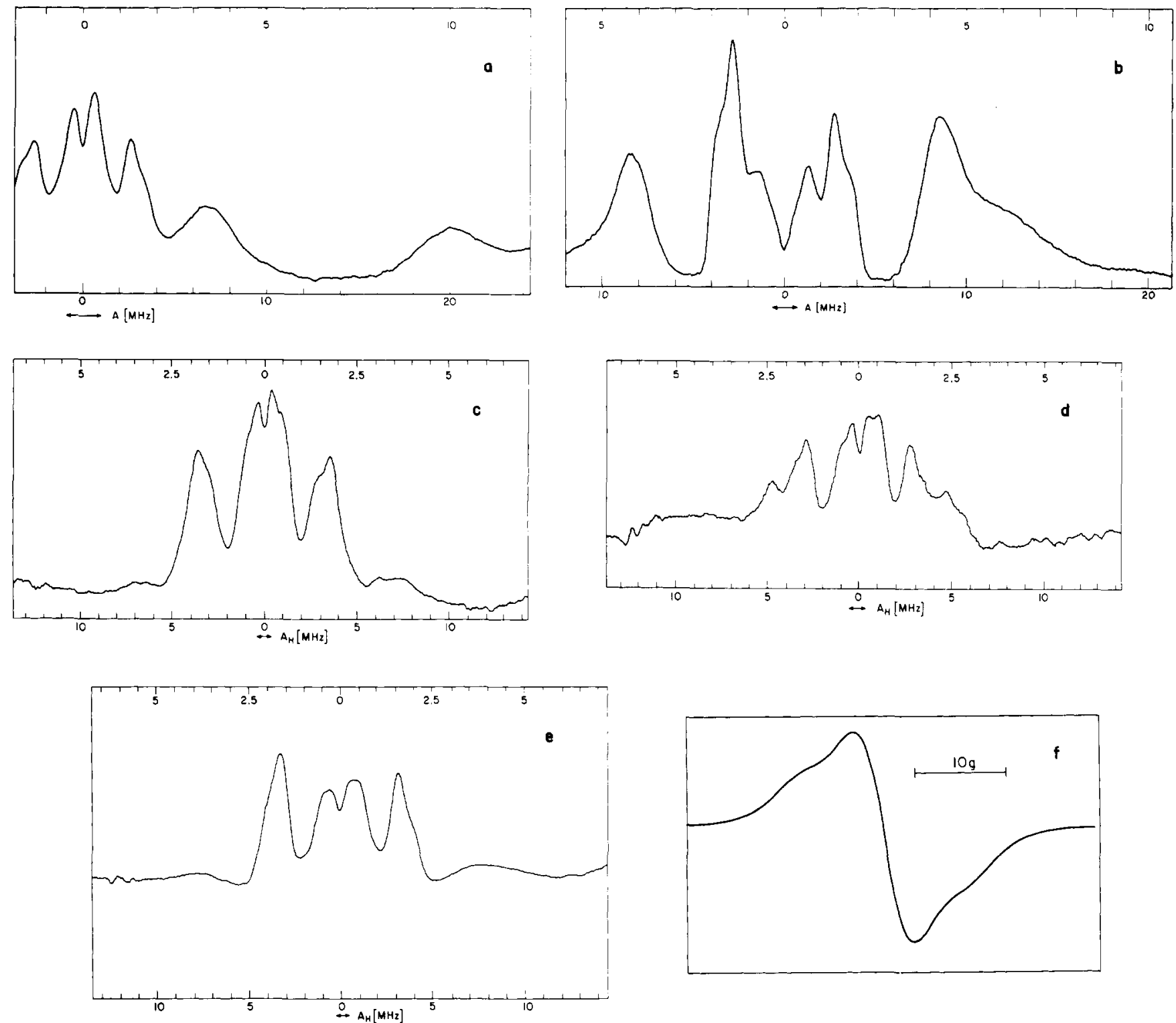

Figure 2. ENDOR spectra of (a) rac-7,8-cis-meso-methylpyrochlorophyllide $a$ (11), (b) rac-9-deoxo-meso-methylpyrochlorophyllide $a$ (10), (c) chlorin $\mathrm{e}_{6}$-TME-Mg (14), (d) rhodochlorin-DME-Mg (13), (e) isochlorin e $\mathrm{e}_{4}$-DME-Mg (15), (f) ESR spectrum of rhodochlorin-DME-Mg (13). (See ENDOR spectrum in Figure 2d.) For convenience the lower scale of each ENDOR spectrum is labeled such that coupling constants of each peak $A$ can be read directly in $\mathrm{MHz}$. Upper scale of each spectrum is labeled in true units of $\mathrm{MHz}$ frequency shift from the free proton resonance value. In other words one unit of the upper scale corresponds to two units of the lower scale. Although these are typical ENDOR spectra for these compounds and are judged suitable for display purposes, it should be noted that the nonlinear aspects of ENDOR spectroscopy are important when comparing Figure 2 with Table I. Table I represents the results of many ENDOR spectra and is thus more complete.

siderations: The dihedral angle of the $10-\mathrm{H}$ bond with the aromatic carbon is fixed and fairly large $\left(\sim 60^{\circ}\right)$; the 10 - $\mathrm{H}$ is in a $\beta$-position not only to the $\gamma$-methine $\mathrm{C}$ atom but also to $\mathrm{C}-10$, which is conjugated to the aromatic system. The rigidity of the fused rings III and V may be held responsible for the poor relaxation and thus for the low intensity of the ENDOR resonance.

Asymmetry of the ENDOR Spectrum. The very different magnitude of the $5-\mathrm{CH}_{3}$ coupling constant and the 1,3- $\mathrm{CH}_{3}$ coupling constants is one of the principal results of this study. It indicates a pronounced asymmetry of the spin density distribution in the $\pi$ cation free radical. A series of compounds was investigated to evaluate the origin of this rather unexpected asymmetry.

The cation radical of $\mathrm{BChl} a(\mathbf{1 6})$ shows a similar difference for the aromatic $\mathrm{CH}_{3}$ coupling constants. As compound 16 has conjugated carbonyl groups in both the 2-and 6-positions, the asymmetry in coupling constants does not arise from the 9-CO group in the chlorophylls. Furthermore, it does not arise from the presence of a 2-vinyl group, as compound 12 that lacks this substituent shows the same basic ENDOR features.

These conclusions are supported by the ENDOR spectrum of the 9-deoxochlorophyllide (10) (Figure 2b), which lacks the 9-CO as well as the 2-vinyl group. The influence of the latter was shown to be small, thus any spectral changes must be due mostly to the 9-CO group. The ENDOR spectrum shows still the same general features as that of $\mathrm{Chl} a \cdot{ }^{+}$, but two changes are noticeable: Both the $A_{2}$ and $A_{4}$ resonances are strongly increased in intensity. Although a detailed interpretation of these changes is not possible without examination of compounds with additional structural modifications, we can make two deductions. First, there is some redistribution in spin density, and second, the asymmetry of the spin distribution persists or is even rather enhanced. Thus, the coupling constant ratio of the two methyl resonances serving as labels for the asymmetry is increased from 2.5 to 2.85 . It should be men- 
tioned that reduction of the 9-CO group leads to a higher overall $\pi$-electron density in the macrocycle, as judged by the ${ }^{1} \mathrm{H}$ NMR ring current induced shifts. This increase seems to be true for the highest occupied MO likewise, as most discernible coupling constants in $\mathbf{1 0}$ are increased as compared to 1,9 , and 12.53

In the compounds without the isocyclic five-membered ring, the two methyl peaks in the ENDOR spectra gradually move together, as the steric hindrance between the substituents at $\mathrm{C}-6, \mathrm{C}-\gamma$, and $\mathrm{C}-7$ is relieved. Chlorin $\mathrm{e}_{6}$ (14) (ENDOR spectrum in Figure $2 \mathrm{c}$ ) has an $\mathrm{A}_{4} / \mathrm{A}_{2}$ ratio of 1.2. The ratio is 1.3 in the rhodochlorin (13) (ENDOR spectrum in Figure 2d), and in the isochlorin $e_{4}$ (15) (ENDOR spectrum in Figure 2e) both methyl group signals have merged. As the coupling constants are in first order related by the same $Q$ value (see below) to the spin densities at the neighboring $\mathrm{C}$ atom, these results clearly indicate that the strongest asymmetric perturbation of the spin density in the chlorophylls is due to the presence of the isocyclic ring. In addition to the effects on the methyl resonances, pronounced changes in other signals in the three ring $\mathrm{V}$ opened compounds can be observed. The $\mathrm{A}_{5}$ resonance assigned to the 7,8-proton couplings is decreased, ${ }^{54}$ and the $A_{1}$ ENDOR line shows resolved fine structure. All of our results confirm the major influence of ring $\mathrm{V}$ on the spin distribution. It is known that the presence of ring $\mathrm{V}$ gives rise to major structural distortions in the vicinity, especially in that of ring III. ${ }^{55}$ While UV-visible and ${ }^{1} \mathrm{H}$ NMR are comparatively unaffected by this steric effect, the configuration of the highest occupied MO is obviously strongly affected by it. It has been argued that there are two such orbitals narrowly spaced in the porphyrin cations ${ }^{56}$ (see below) and the observed changes are possibly due to a different degree of mixing between the two orbitals.

The ESR Line Width. The peak-to-peak ESR line width $\Delta H$ of an inhomogeneously broadened Gaussian line is related to the second moment $\left\langle\omega^{2}\right\rangle$ by $\Delta H^{2}=4\left\langle\omega^{2}\right\rangle$. In the case of chlorophyll, coupling with more than one kind of nucleus can occur, and the second moment can be split up into several components

$$
\begin{aligned}
\left\langle\omega^{2}\right\rangle=\left\langle\omega^{2}\right\rangle_{{ }_{H}}+\left\langle\omega^{2}\right\rangle_{13} \mathrm{C}+\left\langle\omega^{2}\right\rangle_{14} \mathrm{~N} & \\
& +\left\langle\omega^{2}\right\rangle_{25} \mathrm{Mg}+\left\langle\omega^{2}\right\rangle_{g \text {-anis }}
\end{aligned}
$$

corresponding to the contributions of the nuclei present and a possible small contribution arising from $g$-factor anisotropy

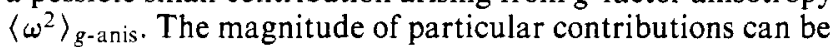
determined by isotope substitution. In the case of hydrogen,

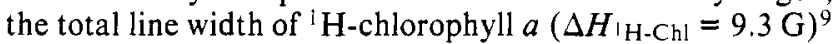
and ${ }^{2} \mathrm{H}$-chlorophyll $a\left(\Delta H_{2} \mathrm{H}-\mathrm{Chl}=3.8 \mathrm{G}\right)^{9}$ is given by

$$
\begin{aligned}
& 1 / 4 \Delta H^{2}{ }_{1 \mathrm{H}-\mathrm{Ch} l}=\left\langle\omega^{2}\right\rangle_{{ }_{\mathrm{H}-\mathrm{Chl}}}=\left\langle\omega^{2}\right\rangle_{{ }_{\mathrm{H}}}+\left\langle\omega^{2}\right\rangle_{\text {rest }} \\
& 1 / 4 \Delta H^{2}{ }_{2 \mathrm{H}-\mathrm{Chl}}=\left\langle\omega^{2}\right\rangle_{{ }^{2} \mathrm{H}-\mathrm{Chl}}=\left\langle\omega^{2}\right\rangle_{{ }^{2} \mathrm{H}}+\left\langle\omega^{2}\right\rangle_{\text {rest }}
\end{aligned}
$$

With the relation

$$
\left\langle\omega^{2}\right\rangle_{{ }_{H}}=15.9\left\langle\omega^{2}\right\rangle_{{ }^{2} \mathrm{H}}
$$

a ${ }^{1} \mathrm{H}$ contribution of $19.2 \mathrm{G}^{2}$ to the total second moment of $21.6 \mathrm{G}^{2}$ has been determined from fully $(\geqslant 99 \%){ }^{1} \mathrm{H}$ - or ${ }^{2} \mathrm{H}$-chlorophylls. ${ }^{57}$

In the case of protons, the second moment is related to the individual coupling constants $A_{i}$ by

$$
\left\langle\omega^{2}\right\rangle=1 / 4 \sum_{i} I_{i} A_{i}^{2}
$$

where $I_{i}$ is the number of equivalent nuclei. With the coupling constants derived from the ENDOR spectra (Table II) a calculated second moment of $\left\langle\omega^{2}\right\rangle=15.6 \mathrm{G}^{2}$ is obtained. This value is in good agreement with experiment, especially when $80 \%$ of the ${ }^{1} \mathrm{H}$ second moment is due to coupling with just five
Table II. Comparison of Experimental Coupling Constants $A$ with Predicted Spin Density Values $\rho^{a}$

\begin{tabular}{llcccr}
\hline & $A, \mathrm{G}$ & $N A^{2}, \mathrm{G}^{2 b}$ & $|\rho|^{c}$ & \multicolumn{1}{c}{$\rho^{d}$} & \multicolumn{1}{c}{$\rho^{e}$} \\
\hline $1-\mathrm{CH}_{3}$ & 1.0 & 3.0 & 0.030 & 0.046 & 0.040 \\
$2 \mathrm{a}-\mathrm{H}$ & 0.14 & 0.02 & 0.005 & & 0.014 \\
$2 \mathrm{~b}-\mathrm{H}_{\mathrm{A}, \mathrm{B}}$ & 0.14 & 0.04 & 0.005 & & -0.023 \\
$3-\mathrm{CH}_{3}$ & 1.31 & 5.18 & 0.039 & 0.032 & 0.004 \\
$4-\mathrm{CH}_{2}$ & 1.0 & 2.0 & 0.030 & 0.032 & 0.008 \\
$5-\mathrm{CH}_{3}$ & 2.67 & 21.4 & 0.080 & 0.020 & 0.070 \\
$10-\mathrm{H}$ & 0.60 & 0.36 & 0.018 & & \\
$7-\mathrm{H}$ & 3.89 & 15.11 & 0.116 & 0.114 & 0.035 \\
$8-\mathrm{H}$ & 3.89 & 15.11 & 0.116 & 0.114 & 0.037 \\
$\alpha-\mathrm{H}$ & 0.18 & 0.03 & 0.007 & -0.043 & -0.067 \\
$\beta-\mathrm{H}$ & 0.18 & 0.03 & 0.007 & -0.043 & -0.073 \\
$\delta-\mathrm{H}$ & 0.18 & 0.03 & 0.007 & +0.017 & -0.005 \\
\hline
\end{tabular}

${ }^{a}$ Experimental values from Table $\mathrm{I}$ using $A$ (in $\mathrm{G}$ ) $=A$ (in $\mathrm{MHz} / 2.83 .{ }^{b} N A^{2} / 4$ is the contribution of a particular group of protons to the total second moment where $N$ is the degeneracy of the coupling constant $A$. This column sums to the value of $62.31 \mathrm{G}^{2}$ and thus accounts for $15.6 \mathrm{G}^{2}$ of the second moment. ' Spin density $\rho$ is obtained from the respective coupling constants by using the following $Q$ values: $Q=27 \mathrm{G} /$ spin for $\alpha$-protons, $Q=33.5 \mathrm{G}$ for $\beta$-protons in agreement with the interpolated value in text. In the case of fixed $\beta$-protons this corresponds to an approximate dihedral angle of $45^{\circ}$. $d$ Private communication from R. Felton. ${ }^{e}$ Private communication from P. S. Song. For ease of comparison Song's $\rho$ values have been "normalized" by division by 5 before listing in Table II.

protons, i.e., the three 5-methyl protons and two at $\mathrm{C}-7$ and C-8. The coupling with the $5-\mathrm{CH}_{3}$ gives rise to a well-defined ENDOR line, but the couplings with $7-\mathrm{H}$ and $8-\mathrm{H}$ give rise to broad and partially hidden lines. These two couplings contribute $44 \%$ of the line width, ${ }^{59}$ and thus small errors in the magnitude of these coupling constants give rise to a considerable error in the second moment. Thus, an increase in each of the 7,8 coupling constants by $0.5 \mathrm{MHz}$ (which may well be a possibility because of the weak, broad ENDOR lines from these protons) would be sufficient to fit the data completely. Although the assignment of the major coupling constants has been done in a straightforward way and is well-supported, the reader is cautioned that resonances not directly accessible may require additional data before the assignments can be considered conclusive. ${ }^{60}$

Spin Density. Proton-electron coupling constants $A_{i}$ are related to the spin density $\rho$ at the carbon atom bearing the proton ( $\alpha$-type coupling) or on the neighboring carbon atom ( $\beta$-type coupling) by a McConnell ${ }^{61}$ relation:

$$
A_{i}=Q \rho_{i}
$$

The spin densities in Table II were obtained from the respective coupling constants by using the following $Q$ values: $Q=27$ $\mathrm{G} /$ spin for $\alpha$-protons, $Q=38 \mathrm{G} /$ spin for rotating $\beta$-protons, and $Q=-9.2 \mathrm{G}+96.7 \mathrm{G} \cos ^{2} \theta$ for fixed $\beta$-protons. The latter formula is derived from ESR measurements on alkylbenzene cation radicals, ${ }^{62}$ where $\theta$ is the dihedral angle between the $\pi$ orbital at $\mathrm{C}_{\alpha}$ and the $\mathrm{C}_{\beta}-\mathrm{H}_{\beta}$ bond. By averaging this relation over $\theta$, the $Q$ value of $38.6 \mathrm{G} / \mathrm{spin}$ for freely rotating $\beta$-protons was obtained. This value obviously represents an upper limit. $Q$ shows a pronounced charge dependence ${ }^{63}$ and decreases with increasing negative charge. The lower limit would then be defined by $Q=25 \mathrm{G} / \mathrm{spin}$ found for anion radicals of alkylbenzenes. ${ }^{64}$ In the alkylbenzenes, the charge is distributed over a much smaller number of $\pi$ centers than in the porphyrins. Assuming an equal charge distribution, the average charge density in the chlorophyll macrocycle is about $1 / 4$ of that in benzene radical ions. From a linear interpolation between the two extremes, a $Q$ value of about $33.5 \mathrm{G} / \mathrm{spin}$ can then be estimated for the Chl $a{ }^{+}$cation radical. 
The results indicate a very low spin density at the methine bridge and intermediate spin density at the $\beta$-pyrrole carbon atoms of rings I, II, and III and the $\alpha$-pyrrole carbons of ring IV. Systematic studies of porphyrin $\pi$-cation radicals have led to the characterization of two types of radicals which show pronounced differences both in their uv-visible and ESR spectra. ${ }^{56}$ The first group of which ZnTPP. ${ }^{+}$is a typical example has a well-resolved ESR spectrum and a series of overlapping visible absorption bands in the region of 500-700 nm. The second group of which MgOEP.+ is representative has an unresolved, inhomogeneously broadened ESR spectrum at room temperature ${ }^{65}$ and shows a strong absorption band at about $700 \mathrm{~nm}$. Both classes of compounds have been investigated in detail by Fajer et al., ${ }^{65}$ and the experimental findings have been interpreted in terms of two closely spaced highest occupied molecular orbitals, which have different relative energies in the two classes of compounds. In ZnTPP.+ the free electron occupies the $a_{2 u}$ orbital, which has high density at the nitrogen and meso positions. In $\mathrm{ZnOEP}^{+}{ }^{+}$the $\mathrm{a}_{1 \mathrm{u}}$ orbital becomes the highest occupied molecular orbital, which is rotated by $\pi / 4$ with respect to the $\mathrm{a}_{2 \mathrm{u}}$ orbital and has high density at the $\pi$ carbons. Although there is probably some mixing of the two states, the model is a good guide in interpreting the spectral properties of $\pi$-cation radicals of porphyrins.

To first order, the results obtained for porphyrin cations can be applied to chlorin cation radicals as well. Both the observed low-spin density at the methine positions of Chl a.+ and the earlier demonstrated low-spin density at the inner nitrogens are against the free electron residing in an $a_{2 \mu}$ type orbital. This is also indicated from the UV-visible spectrum of $\mathrm{Chl} a^{+}{ }^{+}$, which shows a strong absorption at $662 \mathrm{~nm}$. However, it is important to note that $\mathrm{Chl} a^{+}{ }^{+}$can only be crudely approximated by the symmetric MgOEP.+.

Two open shell calculations for the chlorophyll $a$ cation radical are compared in Table II. In the first study (Table II) the $\mathrm{Chl} a^{+}{ }^{+}$is approximated by a $\mathrm{Mg}$ chlorin. ${ }^{66}$ The $C_{2}$ symmetry of the model is reflected in the equal spin density at symmetric positions. The orbital occupied by the free electron is basically of the $a_{2 u}$ type, but there are pronounced differences in the spin density distribution between rings I and III and ring II, respectively. The net spin density of the rings increases monotonically with increasing distance from the reduced peripheral double bond (IV $<$ I, III $<$ II) and four of the $\beta$-pyrrole carbons $(1,6,3,4)$ gain considerable spin density from the neighboring $\alpha$-pyrrole carbons. A (small) negative spin density is only obtained at the $\alpha$-and $\beta$-methine positions. The more detailed SCF-CI calculation of Song ${ }^{67}$ includes both the 2vinyl and the 6-carbonyl group (Table II). Two pronounced differences in this model are immediately obvious. It yields a much less symmetric spin density distribution, and it gives rise to both large positive and negative spin densities. The total spin in this model is represented by five rather than one spin, which renders an assessment of the spin density from proton ENDOR data alone difficult. The model correctly predicts a much larger (negative) spin density on C-5 than on C-1, but the small spin density on $\mathrm{C}-3$ and the predicted large spin density at the methine positions seems to be incompatible with the ENDOR results. Moreover, the ENDOR results do indicate the asymmetric spin density is due neither to the 2-vinyl nor to the 6-CO substituent (the 9-carbonyl group), but rather to the presence of the isocyclic ring. It is the influence of this ring, however, which is most difficult to take into account in calculations of the spin density in the $\pi$ cation radical. If the sign for the spin densities predicted by the latter calculation (Song) (Table II) is used the total spin density in the $\beta$-pyrrole, the methine, and the vinyl $C$ atoms is about $31 \%$ of one electron. As the four inner nitrogen atoms, as well as the central magnesium atom, are essentially spin free, we are forced to conclude that the remaining spin does reside in the $\alpha$-pyrrole carbons as well as in the 6-carbonyl group.

\section{Conclusions}

Our ENDOR results show protons $7-\mathrm{H}$ and $8-\mathrm{H}$ to have the largest proton-electron coupling constants. As these are fixed $\beta$-protons, small changes in the dihedral angle between them can make a significant change in their coupling constants, and thus in both the ENDOR and ESR line widths. The next largest coupling constant arises from the $5-\mathrm{CH}_{3}$ group, followed by the $1-\mathrm{CH}_{3}, 3-\mathrm{CH}_{3}$, and $4-\mathrm{CH}_{2}$ groups. We have tentatively assigned small coupling constants to all macrocycle $\alpha$-protons, i.e., $\alpha, \beta, \delta$, the 2 -vinyl protons, and the $\beta$ - 10 -H. In the ENDOR experiments described here, $\alpha$-protons are expected to give rise to broad and very weak ENDOR signals, and thus can easily go undetected. Consequently, it is possible that some of these $\alpha$-protons may have a somewhat larger coupling constant than we have indicated. We again caution the reader that we still do not account for a small amount $(\sim 3$ $\mathrm{G}^{2}$ ) in the second moment of the proton-electron hyperfine interactions. As previously mentioned, this missing portion of the second moment may be attributed to inaccuracies in the measurement of the coupling constants of the 7-and 8- (fixed) $\beta$-protons. These have wide ENDOR resonances that make accurate determination of coupling constants difficult. However, we cannot rule out at this time the possibility that some of the missing second moment arises from unobserved $\alpha$-protons that have higher spin density than we have tentatively assigned.

Acknowledgment. This work was carried out under the auspices of the U.S. Energy Research and Development Administration. We gratefully acknowledge the award of a stipend to H.S. by the Deutsche Forschungsgemeinschaft, Bonn Bad-Godesberg. This paper is dedicated to Professor Hans $\mathrm{H}$. Inhoffen on the occasion of his 70th birthday.

\section{References and Notes}

(1) G. Feher, Phys. Rev., 103, 834-835 (1956)

(2) G. Feher, A. J. Hoff, R. A. Isaacson, and J. D. McElroy, Biophys. Soc. Abstr., 13, 6 ta (1973).

(3) J.R. Norris, M. E. Druyan, and J. J. Katz, J. Am. Chem. Soc., 95, 1680-1682 (1973).

(4) G. Feher, A. J. Hoff, R. A. Isaacson, and L. C. Ackerson, Ann. N. Y. Acad. Sci., 244, 239-259 (1975).

(5) J. R. Norris, H. Scheer, and J. J. Katz, Ann. N.Y. Acad. Sci., 244, 261-280 (1975).

(6) J. R. Norris, H. Scheer, M. E. Druyan, and J. J. Katz, Proc. Natl. Acad. Sci. U.S.A., 71, 4897-4900 (1974)

(7) D. C. Borg, "Free Radicals in Biology", W. A. Pryor, Ed., Academic Press, New York, N.Y., 1975, pp 69-147.

(8) D. C. Borg, A. Forman, and J. Fajer, J. Am. Chem. Soc., in press

(9) J. R. Norris, R. A. Uphaus, H. L. Crespi, and J. J. Katz, Proc. Natl. Acad. Sci. U.S.A., 68, 625-628 (1971).

(10) J. D. McElroy, G. Feher, and D. C. Mauzerall, Biochim. Biophys. Acta, 267 363-374 (1972).

(11) J. R. Norris, R. A. Uphaus, and J. J. Katz, Biochim. Biophys. Acta, 275, 161-168 (1972)

(12) J. J. Katz and K. Ballschmiter, Angew. Chem., 7, 286-287 (1968)

(13) J. J. Katz and K. Ballschmiter, Nature (London), 220, 1231-1233 (1968).

(14) J. J. Katz, K. Ballschmiter, M. Garcia-Morin, H. H. Strain, and R. A. Uphaus, Proc. Natl. Acad. Sci. U.S.A., 60, 100-107 (1968).

(15) J. J. Katz and J. R. Norris, "Current Topics in Bioenergetics", D. R. Sanad and L. Packer, Ed., Academic Press, New York, N.Y., 1973, pp 41-75.

(16) F. K. Fong, J. Theor. Biol., 46, 407-420 (1974); Proc. NatI. Acad. Scl U.S.A., 71, 3692-3695 (1974)

(17) F. K. Fong, Appl. Phys., 6, 151-166 (1975)

(18) F. K. Fong and V. J. Koester, J. Am. Chem. Soc., 97, 6888-6890 (1975).

(19) F. K. Fong, J. Am. Chem. Soc., 97, 6890-6892 (1975).

(20) F. K. Fong and V. J. Koester, Biochim. Biophys. Acta, 423, 52-64 (1976).

(21) F. K. Fong and N. Winograd, J. Am. Chem. Soc., 98, 2287-2288 (1976).

(22) N. Winograd, A. Shepard, D. H. Karweik, V. J. Koester, and F. K. Fong, $J$ Am. Chem. Soc., 98, 2369-2370 (1976).

(23) L. L. Shipman, T. M. Cotton, J. R. Norris, and J. J. Katz, Proc. Natt. Acad. Sci. U.S.A., 73, 1791-1794 (1976).

(24) S. G. Boxer and G. L. Closs, J. Am. Chem. Soc., 98, 5406-5408 (1976).

(25) J. S. Hyde, G. H. Rist, and L. E. G. Eriksson, J. Phys. Chem., 72, 4269-4276 
(1968).

(26) H. H. Strain and W. A. Svec, "The Chlorophylls", L. P. Vernon and G. R. Seely, Ed., Academic Press, New York, N.Y., 1966, pp 21-66.

(27) W. A. Svec, "The Porphyrins". D. Dolphin, Ed., in press.

(28) J. B. Conant and J. F. Hyde, J. Am. Chem. SoC., 52, 1233-1239 (1930); H. Fischer and E. Lakatos, Justus Liebigs Ann. Chem., 506, 123-137 (1933).

(29) H. Fischer and H. Gibian, Justus Liebigs Ann. Chem., 550, 208-251 (1942); 552, 153-166 (1942)

(30) H. Fischer and H. Orth, "Die Chemie des Pyrrols", Vol. II, Akademische Verlagsgesellschaft, Leipzig, 1940, reprinted by Johnson Reprint Corp., New York, N.Y., 1968.

(31) We are indebted to Professor H. H. Inhoffen for a generous gift of compounds 5,6 , and 7 .

(32) H. Wolf and H. Scheer, Justus Liebigs Ann. Chem., 1710-1740 (1973).

(33) C. D. Mengler, Dissertation, Technische Hochschule Braunschweig, 1966; H. H. Inhoffen, J. W. Buchler, and P. Jager, Fortschr. Chem. Org. Naturst., 26, 284-355 (1968).

(34) H. Scheer and J. J. Katz, J. Am. Chem. Soc., 97, 3273-3275 (1975)

(35) The insertion of $\mathrm{Mg}$ into pheophorbides containing the $\beta$-ketoester moiety by $\mathrm{Mg}$ complexes of "soft" ligands has been reported recently $(H$. P. Isenring, E. Zass, K. Smith, H. Falk, J.L. Luisier, and A. Eschenmoser, Helv. Chim. Acta, 58, 2367 (1975)). Thus, although a difficult task, in principle this ENDOR work could be repeated on isotopically labeled chlorophyll a (1) instead of methylpyrochlorophyllide a (9).

(36) S. J. Baum, B. F. Burnham, and R. A. Plane, Proc. Natl. Acad. Sci. U.S.A., 52, 1439-1442 (1964).

(37) H. Scheer and H. H. Inhoffen, "The Porphyrins", D. Dolphin, Ed., Academic Press, New York, N.Y., in press.

(38) One of these bands probably originates from a $Q_{x}(0-1)$ transition. The assignment of the second band is uncertain, but has been suggested to be specifically a free-base transition. ${ }^{39}$

(39) C. Weiss, J. Mol. Spectrosc., 44, 37-80 (1972)

(40) G. W. Kenner, K. M. Smith, and M. J. Sutton, Tetrahedron Lett., 1303-1306 (1973).

(41) R. B. Woodward and V. Skaric, J. Am. Chem. Soc., 83, 4676-4678 (1961); R. Bonnett, I. A. D. Gale, and G. F. Stephenson, J. Chem. Soc., C, 1168-1172(1967).

(42) H. Scheer and J. J. Katz, unpublished results.

(43) R. C. Dougherty, H. H. Strain, and J. J. Katz, J. Am. Chem. Soc., 87, 104-109 (1965).

(44) F. C. Pennington, H. H. Strain, W. A. Svec, and J. J. Katz, J. Am. Chem. Soc., 86, 1418-1426 (1964).

(45) G. L. Closs, J. J. Katz, F. C. Pennington, M. R. Thomas, and H. H. Strain, J. Am. Chem. Soc., 85, 3809-3821 (1963); H. Scheer and J. J. Katz, "Porphyrins and Metalloporphyrins", K. M. Smith, Ed., Elsevier, Amsterdam, 1975, pp 399-524.

(46) R. C. Dougherty, H. L. Crespi, H. H. Strain, and J. J. Katz, J. Am. Chem. Soc., 88, 2854-2855 (1966).

(47) J. J. Katz, R. C. Dougherty, H. L. Crespi, and H. H. Strain, J. Am. Chem. Soc., 88, 2856-2857 (1966)

(48) H. Scheer and J. J. Katz, Proc. NatI. Acad. Sci. U.S.A., 71, 1626-1629
(1974).

(49) G. E. Ficken, R. B. Johns, and R. P. Linstead, J. Chem. Soc., 2272-2280 (1956).

(50) I. Fleming, Nature (London), 216, 151-152 (1967)

(51) H. Brockmann, Jr., Justus Liebigs Ann. Chem., 754, 139-148 (1971).

(52) J. Fajer, D. C. Borg, A. Forman, R. H. Felton, D. Dolphin, and L. Vegh, Proc. Natl. Acad. Sci. U.S.A., 71, 994-998 (1974).

(53) In a similar manner, the molecular asymmetry of the highest occupied molecular orbital (if judged by the hyperfine coupling constants), and of the net electron density (if judged by the facile ${ }^{1} \mathrm{H} /{ }^{2} \mathrm{H}$ exchange of the $5-\mathrm{CH}_{3}$ group, and by the ${ }^{1} \mathrm{H}$ NMR shifts ${ }^{32}$ ), respectively, parallel each other in the chlorophylls. Similar effects have been observed recently in hemins (LaMar and co-workers, private communication).

(54) The ESR spectrum of 13 (Figure $2 f$ ) shows structure typical of a large 2 . proton $A$. Such coupling is evidence in some ENDOR spectra at $A=8 \mathrm{MHz}$ (field dependent) but is unclear in Figure $2 f$

(55) R. C. Peterson, J. Am. Chem. Soc., 93, 5629-5634 (1971); J. Gassmann, I. Strell, F. Brandt, M. Sturm, and W. Hoppe, Tetrahedron Lett., 4609-4612 (1971); M. S. Fisher, D. H. Templeton, A. Zalkin, and M. Calvin, J. Am Chem. Soc., 94, 3613-3619 (1972); C. E. Strouse, Proc. Natl. Acad. Sci. U.S.A., 71, 325-328 (1973); L. Kratky and J. D. Dunitz, Acta Crystallogr., Sect. $B, 31,1586-1589$ (1975).

(56) D. Dolphin and R. H. Felton, Acc. Chem. Res., 7, 26-32 (1974).

(57) By similar substitution experiments, a small contribution from naturally abundant ${ }^{13} \mathrm{C}$ and a negligible contribution to ${ }^{25} \mathrm{Mg}$ has been deduced. ${ }^{11,58}$ The remaining line width must then be due to nitrogen interactions with a small $g$-factor anisotropy contribution.

(58) M. E. Druyan, J. R. Norris, and J. J. Katz, J. Am. Chem. Soc., 95, 1682-1683 (1973).

(59) The second moment is calculated here from a stick spectrum, which is certainly not true for the $7-\mathrm{H}$ and $8-\mathrm{H}$ couplings. However, in view of the difficulties in integrating the ENDOR signal this procedure seemed to be reasonable.

(60) After completion of this paper a separate, but not totally independent, determination of the proton-electron hyperfine coupling constants of chlorophyll a cation radical by ${ }^{1} \mathrm{H}$ NMR has been reported (J.K. Sanders and J. C. Waterton, J. Chem. Soc., Chem. Commun., 247-48 (1976)) with a general agreement in the relative magnitudes of the $A$ values. Recent H N NMR results (private communication from Dr. J. K. M. Sanders) now show excellent agreement with our ENDOR method except for $A=3.8$ $\mathrm{MHz}$ for the $\delta$ methine proton. The NMR method requires the ENDOR data for absolute calibration of the hyperfine coupling constants.

(61) H. M. McConnell, J. Chem. Phys., 24, 764-766 (1956).

(62) M. K. Carter and G. Vincow, J. Chem. Phys., 32, 1535-1539 (1960)

(63) G. R. Underwood and V. L. Vogel, J. Chem. Phys., 51, 4323-4326 (1969).

(64) E. DeBoer and J. P. Colpa, J. Phys. Chem., 71, 21-29 (1967)

(65) D. C. Borg, J. Fajer, R. H. Felton, and D. Dolphin, Proc. Nati. Acad. Sci U.S.A., 67, 813-820 (1970); J. Fajer, D. C. Borg, A. Foreman, R. H. Felton, L. Vegh, and D. Dolphin, Ann. N.Y. Acad. Sci. 206, 349-364 (1973).

(66) R. Felton and J. Fajer, private communication.

(67) P. S. Song, private communication.

\title{
Advantages of ${ }^{15} \mathrm{~N}$ NMR in Studying Delocalization and Evaluating the Energy Barriers of $\mathrm{C}-\mathrm{N}$ Rotation Process in Amides, Thioamides, and Related Compounds
}

\author{
G. J. Martin,* J. P. Gouesnard, J. Dorie, C. Rabiller, and M. L. Martin \\ Contribution from the Chimie Organique Physique, E.R.A.-C.N.R.S. 315 , \\ Université de Nantes, 44037 Nantes, France. Received June 30, 1976
}

\begin{abstract}
Linear correlations are shown to exist between the activation energy $E_{\mathrm{a}}$ of the $\mathrm{C}-\mathrm{N}$ rotation process and $\delta^{15} \mathrm{~N}$ in $N, N$-dimethylamino derivatives. The relations are applied to the prediction of $E_{\text {a }}$ values related to high or low energy barriers, where the direct NMR methods of measurement are not applicable. The validity of the correlations is discussed on the basis of semiempirical calculations of the nitrogen chemical shifts.
\end{abstract}

The variation of ${ }^{14} \mathrm{~N}$ chemical shifts in amides and thioamides was qualitatively explained by Hampson and Mathias ${ }^{1}$ in terms of the delocalization of the nitrogen lone pair. Siddall et al., ${ }^{2}$ however, failed to observe the predicted correlation between ${ }^{14} \mathrm{~N}$ downfield shifts and the increase of barrier heights to rotation in $\mathrm{N}$-alkyl substituted amides and explained their results by the prominent role of steric effects. We wish to present here ${ }^{15} \mathrm{~N}$ data which provide a good means of evaluating $\mathrm{C}-\mathrm{N}$ bond rotation energy barriers in cases where conventional dynamic NMR methods (line shape analysis or Forsen-Hoffmann double resonance studies) are not applicable. Our results also indicate a striking difference in the behavior of ${ }^{13} \mathrm{C}$ and ${ }^{15} \mathrm{~N}$ chemical shifts in a $>\mathrm{N}=\mathrm{C}<$ fragment, insofar as electronic delocalization is concerned.

Table I collects $\delta^{15} \mathrm{~N}$ and $\delta^{13} \mathrm{C}$ values for the $>\mathrm{N}=\mathrm{C}<$ fragment of selected compounds for which good activation energy values are available. Amides, thioamides, and related compounds were considered and good correlations between $E_{\mathrm{a}}$ 